\title{
Passive scalar diffusion in and above urban-like roughness under weakly stable and unstable thermal stratification conditions
}

\author{
Isao Kanda ${ }^{\mathrm{a}}$, Yukio Yamao ${ }^{\mathrm{b}}$ \\ ${ }^{a}$ Faculty of Agriculture, Ehime University, 3-5-7 Tarumi, Matsuyama, Ehime, 790-8566, Japan \\ ${ }^{b}$ National Institute for Environmental Studies, 16-2 Onogawa, Tsukuba, Ibaraki, 305-8506, Japan
}

\section{Abstract}

Wind-tunnel experiments were conducted to investigate passive scalar diffusion behaviour in and above urban-like roughness under various thermal stratification conditions. Staggered arrays of rectangular blocks with aspect ratios of 0.96 or 1.92 were used to model the urban canopies. Weakly stable and unstable thermal stratification conditions were created by controlling the temperature of the windtunnel floor and the airflow. Above the modelled urban roughness, vertical profiles of the velocity and temperature were measured by a laser Doppler anemometer and a cold-wire thermometer, respectively. The estimated Obukhov length was found comparable or larger than the boundary-layer thickness, indicating very weak stratification. Passive-scalar diffusion was studied by emitting ethane gas from a point source on the wind-tunnel floor. Thermal stratification had substantial effect on diffusion even in the vicinity of the source where mechanical mixing by the model blocks was presumed to eliminate the stratification effect.

Keywords: Diffusion, Surface roughness, Thermal stratification, Urban canopy, Wind tunnel

\section{Introduction}

In urban air pollution problems, prediction of pollutant diffusion from emission sources is important. Many numerical models for diffusion prediction have 4 been developed, but such models need to be validated against field or laboratory 
data. In atmospheric diffusion, wind-tunnel experiments provide validation data under controlled conditions.

In the real atmosphere, thermal stratification usually occurs and affects the diffusion behaviour. Although mechanical mixing is considered to reduce the stratification effect in urban geometries, many wind-tunnel experiments revealed non-negligible effects of stratification. Representative studies are reviewed in the following. Experiments over smooth surfaces with small values (much less than the threshold 1-5 proposed by Robins 2003) of the roughness Reynolds number are omitted.

Shirakata et al. (2002) used various vorticity generators and roughness elements to generate turbulence and stratification profiles that reproduced diffusion behaviour observed in the field for the Pasquill-Meade stability classes (Pasquill 1961; Meade 1959). Hort and Robins (2002) studied diffusion from the top of cylindrical tanks over a rough surface under neutral and stable stratification conditions. Similarly, Yassin (2013) simulated diffusion from a stack on an isolated building immersed in a boundary layer over a rough surface under neutral, stable, and unstable stratification conditions. Robins et al (2001) studied the diffusion of dense gas over a rough surface under stable stratification conditions. Their study provided a more detailed characterization of the turbulence field than did other studies involving diffusion. Uehara et al. $(1997,1998,1999)$ studied recirculation and diffusion in street canyons under neutral, stable, and unstable stratification conditions. The street canyons they studied were not two-dimensional, but were located between the walls of buildings on opposite sides of a corridor along an inline array of simulated three-dimensional buildings. Xie et al. (2013) have reported both numerical models and wind-tunnel results simulating the boundary layer over arrays of buildings, but they provided little information about the windtunnel experiments. Uehara et al. (2007) conducted simulations of diffusion from line sources in a reduced-scale model of a real urban area under neutral, stable, and unstable conditions, but their comparison with field observations was confined 
to results under neutral stratification conditions. Yassin et al. (2005) conducted a similar investigation of diffusion from point sources under various stability conditions.

Field experiments using large modelled building arrays have been reported (Inagaki and Kanda 2008, 2010; Yee and Biltoft 2004; Gailis and Hill 2006). However, the inevitable variability of meteorological conditions and the limited number of measurement locations have made it difficult to draw general conclusions.

This paper presents a wind-tunnel study of passive scalar diffusion from a ground source in modelled urban geometries under neutral, stable, and unstable ambient thermal stratification conditions. The ground-level emission assumes tailpipe emission from road vehicles, especially hot spots at road crossings. The surface roughness is the same as in Kanda et al. (2011) except that their realcity models are replaced with arrays of parallelpiped blocks. Kanda et al. (2011) demonstrated that the results of wind-tunnel diffusion experiments and field monitoring agreed well for four different urban areas under neutral stratification condition. In effect, this paper adds to Kanda et al. (2011) thermal stratification as another parameter affecting diffusion.

\section{Experimental methods}

The experiments were conducted in the Atmospheric Diffusion Wind Tunnel at the National Institute for Environmental Studies in Japan (Fig. 1; Ogawa et al. 1981). The wind tunnel was of the closed-circuit type, with a test section $24 \mathrm{~m}$ long, $3.0 \mathrm{~m}$ wide, and about $2.2 \mathrm{~m}$ high. The wind speed $U_{0}$ at the test-section entrance was fixed at $1.5 \mathrm{~m} \mathrm{~s}^{-1}$. A higher wind speed, such as $U_{0}=3.5 \mathrm{~m} \mathrm{~s}^{-1}$ adopted by Kanda and Yamao (2011) (hereinafter denoted as KY), was desirable for the purpose of securing a fully turbulent boundary-layer flow, but to create distinctly different stratification conditions within the capacity of the wind tunnel, a lower speed was required. We note that the chosen wind speed of $U_{0}=1.5 \mathrm{~m} \mathrm{~s}^{-1}$ 


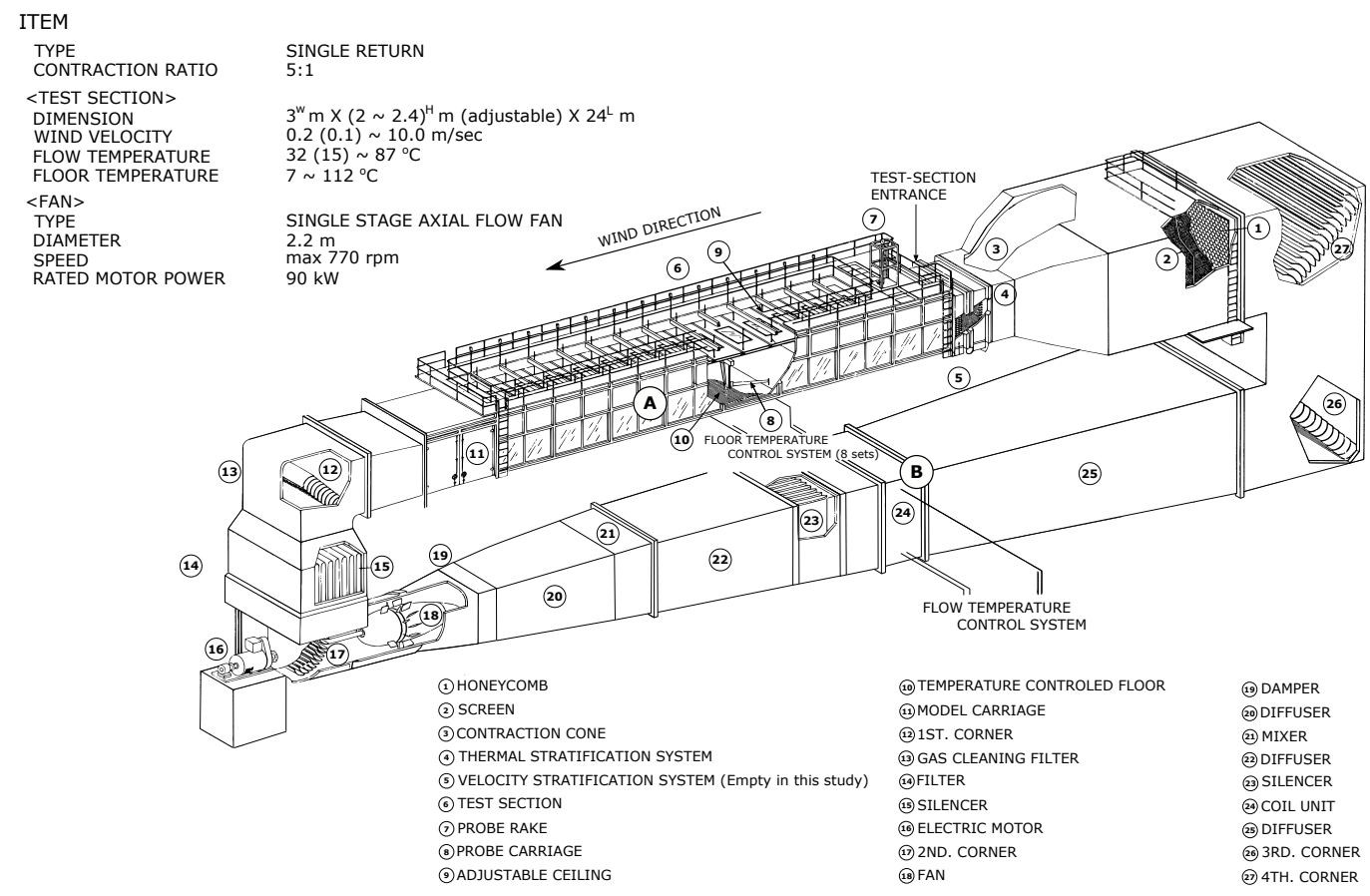

Figure 1: Overview of the wind tunnel. The test-section size is $24 \mathrm{~m}$ long, $3.0 \mathrm{~m}$ wide, and about $2.2 \mathrm{~m}$ high. The seeding particles for the laser Doppler anemometry were generated at " $\mathrm{A}$ " for the neutral and unstable cases, and at "B" for the stable cases. A higher-resolution figure can be found in Ogawa et al. (1981). 
was a little above a threshold $U_{0}$ of $1.0 \mathrm{~m} \mathrm{~s}^{-1}$, below which the time-averaged properties of the wake flow behind a two-dimensional hill model ( $63^{\circ}$ slope angle) depended on $U_{0}$ under neutral stratification conditions (Kanda et al. 2013). As described in KY, the downwind acceleration of the flow due to the boundary-layer growth was expected to have negligible effect on the experimental results. The coordinates $(x, y, z)$ were defined such that $x$ was the windward coordinate with its origin at the source described later in this section, $y$ was the horizontal crosswind coordinate with its origin at the centre line of the wind tunnel, and $z$ was the vertical coordinate with its origin at floor level.

Thermal stratification was created by controlling the temperature of the recirculating air and that of the $3 \times 3-\mathrm{m}$ floor panels in the test section. The temperature $T_{\infty}$ of the recirculating air was measured at the mid-height of the test-section entrance, and the temperatures $T_{\mathrm{f}}$ of the floor panels were measured at 6 evenly spaced points on each panel by thermocouples embedded immediately below the top surface of the panel. In the cases of unstable stratification, the floor panels were heated to $40^{\circ} \mathrm{C}$ and the recirculating air was maintained at $15^{\circ} \mathrm{C}$. In the cases of stable stratification, the floor panels were cooled to $15^{\circ} \mathrm{C}$ and the recirculating air was heated to $40^{\circ} \mathrm{C}$. The upper temperature $40^{\circ} \mathrm{C}$ was chosen so that the secondary circulation induced by the temperature difference on the walls and the ceiling was negligibly weak and the glycerine mist for the velocity measurement did not evaporate while flowing through the heat exchanger in the underground section of the wind tunnel. The neutral cases were conducted in the winter, and the air-conditioning of the wind-tunnel building maintained both the floor panels and the recirculating air at $16^{\circ} \mathrm{C}$. On both sides of the test section, outer 1-m tall and inner $0.45-\mathrm{m}$ tall partitions were installed to isolate the experimental area from the secondary circulation (Ogawa et al. 1981). The effective width of the test-section between the inner partitions was therefore about $1.8 \mathrm{~m}$. Because the width was only twice as large as the boundary-layer thickness, there could have been some crosswind variation in the flow properties. 
Table 1: Experimental conditions and parameter values. The bulk Richardson number $R i_{\mathrm{b}}$ is defined by $R i_{\mathrm{b}}=19.6\left(T_{\infty}-T_{\mathrm{f}}\right) \delta /\left(T_{\infty}+T_{\mathrm{f}}\right) U_{\infty}^{2}$.

\begin{tabular}{lcccccc}
\hline stability & \multicolumn{2}{c}{ Stable } & \multicolumn{2}{c}{ Neutral } & \multicolumn{2}{c}{ Unstable } \\
\hline$T_{\infty}\left({ }^{\circ} \mathrm{C}\right)$ & \multicolumn{2}{c}{4} & \multicolumn{2}{c}{16} & \multicolumn{2}{c}{15} \\
$T_{\mathrm{f}}\left({ }^{\circ} \mathrm{C}\right)$ & \multicolumn{2}{c}{40} & \multicolumn{2}{c}{16} & \multicolumn{2}{c}{40} \\
$\delta(\mathrm{m})$ & \multicolumn{2}{c}{15} & \multicolumn{2}{c}{16} & \multicolumn{2}{c}{$0.8-0.9$} \\
$H(\mathrm{~mm})$ & $0.6-0.7$ & $0.8-0.9$ & 0.8 & 96 \\
$U_{\infty}\left(\mathrm{m} \mathrm{s}^{-1}\right)$ & 48 & 96 & 48 & 96 & 48 & 96 \\
$u_{*}\left(\mathrm{~m} \mathrm{~s}^{-1}\right)$ & 1.60 & 1.64 & 1.59 & 1.62 & 1.58 & 1.61 \\
$u_{*} / U_{\infty}$ & 0.070 & 0.090 & 0.104 & 0.116 & 0.128 & 0.146 \\
$T_{*}(\mathrm{~K})$ & 0.044 & 0.055 & 0.065 & 0.072 & 0.081 & 0.091 \\
$L_{\text {ОВ }}(\mathrm{m})$ & 0.54 & 0.47 & - & - & -0.86 & -0.59 \\
$R i_{\mathrm{b}}(\mathrm{m})$ & 0.7 & 1.4 & - & - & -1.4 & -2.7 \\
$\partial T / \partial z(z=H / 2)\left(\mathrm{K} \mathrm{m}^{-1}\right)$ & 68 & 50 & - & - & -29 & -24 \\
\hline
\end{tabular}

Two types of roughness elements were placed on the wind-tunnel floor, one in the upwind part and the other in the downwind part. The upwind roughness elements consisted of flat aluminium bars fitted with vertical rectangular plates (Fig. 2 in KY). The height $h$ from the base of the bar to the top of the plates was either 20 or $40 \mathrm{~mm}$. The bars were placed at $0.2-\mathrm{m}$ intervals in the $x$ direction, and the plates were placed in a staggered formation (the two rows of dashed lines toward the left edge of Fig. 2 represent the last two bars). In the following discussion, upwind roughness is denoted by the height in centimetres, i.e., h2 and h4 for roughness elements with a height $h$ of $2 \mathrm{~cm}$ and $4 \mathrm{~cm}$, respectively.

The downwind roughness element was an urban-canopy model consisting of rectangular wooden blocks with a square horizontal cross section, a horizontal side length $L$ of $50 \mathrm{~mm}$, and a height $H$ of 48 or $96 \mathrm{~mm}$. The number of test cases was six, as indicated in Table 1. For the 48-mm blocks, the upwind rough- 


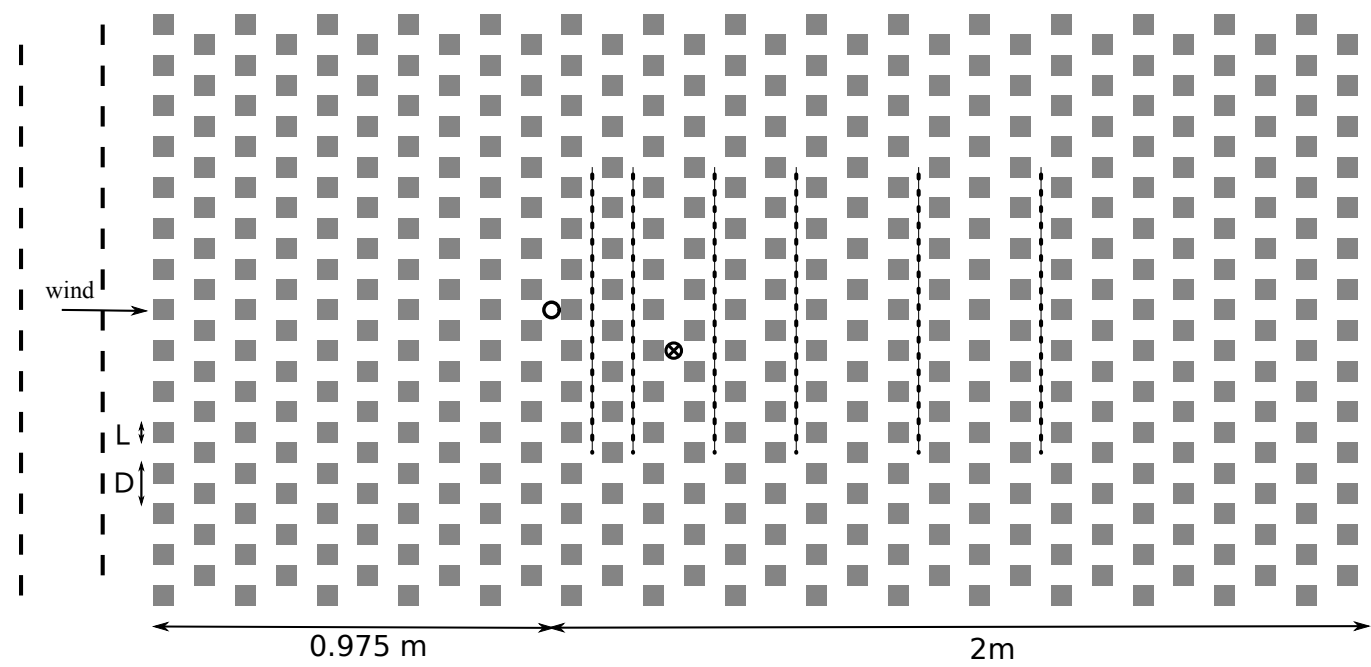

Figure 2: Plan view of the urban canopy model. The grey squares represent the blocks making up the canopy model. The long-dash lines upwind of the canopy model represent the vertical plates that are the roughness elements. The circled cross indicates the point where the vertical velocity profiles were measured. The open circle indicates the location of the source on the wind-tunnel floor. The plume concentration was measured at the crosswind sections indicated by the short-dash lines. $L$ and $D$ indicate the length of a block side and the length of a side of a lot containing one block, respectively.

ness was $\mathrm{h} 2$, and for the $96-\mathrm{mm}$ blocks, the upwind roughness was $\mathrm{h} 4$. These combinations were associated with the least fetch required for adjustment of the turbulence properties after the roughness change investigated in KY. The plan area ratio $\lambda_{\mathrm{p}}$ was defined by

$$
\lambda_{\mathrm{p}}=\frac{L^{2}}{D^{2}},
$$

where $D^{2}\left(0.01 \mathrm{~m}^{2}\right)$ was the lot area per block. The value of $\lambda_{\mathrm{p}}$ was therefore 0.25 . The wooden blocks were arranged in a staggered formation as shown in Fig. 2. The blocks were placed piece-by-piece on the wind-tunnel floor panels so that the floor panels whose temperatures were controlled to within $\pm 1^{\circ} \mathrm{C}$ by the builtin water jacket, were exposed to the ambient air in places where there were no blocks. The blocks were laid on the previous day of each experimental run, and the thermal condition in the wind tunnel was made to stabilize for at least two hours after activating the heat exchange system and air flow. The surface temperature of 
the blocks was not measured although the blocks were expected to have been in a thermal equilibrium with the ambient air during the measurements. The roughness Reynolds number $R e_{*}$ based on the roughness length $3.4 \mathrm{~mm}$ estimated in KY was larger than 15, and hence the flow over the roughness elements could be regarded as sufficiently turbulent.

Immediately downwind of the test section entrance, a 0.18-m high castellated fence and five 0.9-m high Counihan-type spires (Counihan 1969; Robins 1979) were installed (Fig. 3). The large eddies generated by these elements increased the thickness of the boundary layer to a little below the height of the spires. Because the large eddies decayed slowly downwind, the boundary layer was inherently in transition. Fully developed boundary layers could have been created without the spires, but the spires were installed because one of the purposes of this study was to characterize the turbulence field under similar geometrical and thermal conditions to those employed in the previous studies conducted in the same wind tunnel (Uehara et al. 1997, 1998, 1999, 2000, 2007; Kanda et al. 2011, 2013).

Although the spires were used to enhance the boundary-layer thickness, it would still be a concern if the development distance to the test area was sufficient particularly in the stable-stratification cases. Detailed downwind develpment could not be measured in our study due to the limited stretch of the optical fibers for the laser Doppler anemometry, but past studies support that our distance $\approx 11 \mathrm{~m}$ was sufficient. In the meteorological wind tunnel at Colorado State University, Ohya et al. (1997) found that the streamwise homogeneity was achieved approximately at $23.5 \mathrm{~m}$ from the entrance of the test section (1.8-m wide and 1.8$\mathrm{m}$ high) of which the floor was cooled from $12.2 \mathrm{~m}$ downwind of the entrance in order to establish stable stratification comparable to or stronger than our cases. In a different wind tunnel at Kyushu University, Ohya (2001) reported that boundary layers were fully grown at $9 \mathrm{~m}$ from the entrance of the test section $(1.5-\mathrm{m}$ wide and 1.2-m high).

The turbulent velocity field was measured with a three-channel laser Doppler 
(a)

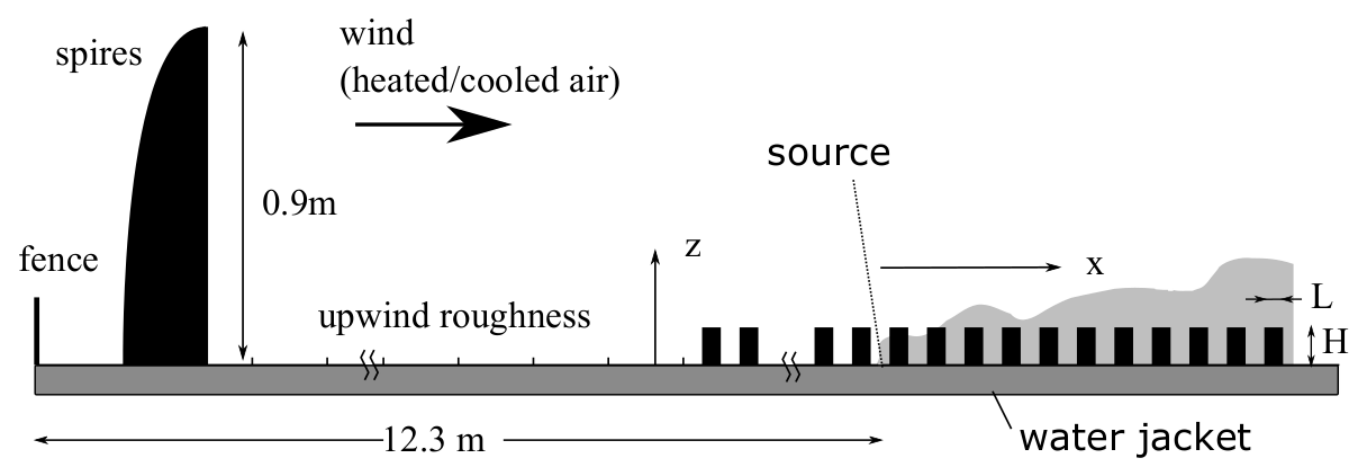

(b)

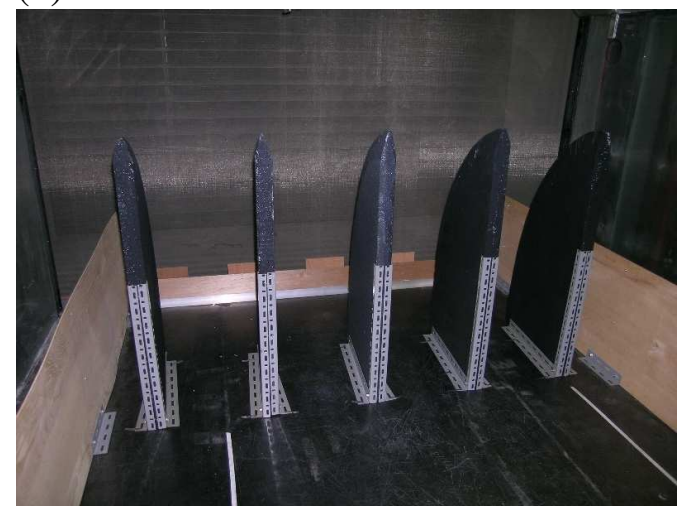

(c)

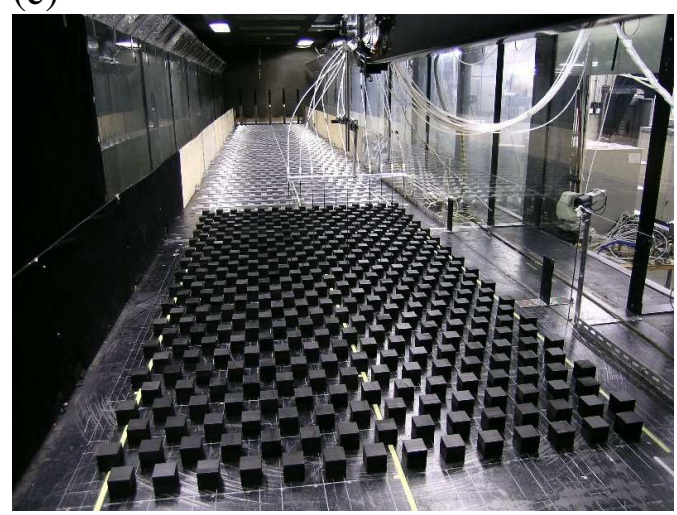

Figure 3: Wind-tunnel test section. (a) Side-view sketch. The vertical:horizontal scaling is 2:1. (b) Spires and fence. (c) Bird's-eye view from a downwind location. 
anemometer (LDA) (Dantec Co., Ltd. in Denmark). Details of the measurement techniques are given in KY. The vertical profiles of velocity were measured at a point located a distance $3 D$ downwind of the source (described later) and immediately behind a block (see Fig. 2). Profiles in the region upwind of the canopy model are presented in KY for the neutral cases but were not measured in this study for the stable and unstable cases. It should be noted that the alignment of the laser beams was adjusted every time a different thermal condition was set up to compensate for the distortion of the metal frame holding the laser heads. The components in the $(x, y, z)$ direction of the mean and perturbation velocity are denoted by $(U, V, W)$ and $(u, v, w)$, respectively. The standard deviation of $(u, v, w)$ is denoted by $\left(\sigma_{u}, \sigma_{v}, \sigma_{w}\right)$. Approximate uncertainty range of the mean velocity value was $0.05 \mathrm{~m} \mathrm{~s}^{-1}$. The seeding particles were a mist of glycerine generated by evaporating liquid glycerine and circulating the condensed particles in the wind tunnel. This seeding method required a large volume of glycerine and risked damage to the ball bearings of the main fan, but enabled accurate measurement of velocity fluctuations of all the length scales existing in the wind tunnel. The location of mist generation was immediately downwind of the urban canopy model in the neutral and unstable cases (A in Fig. 1) and immediately downwind of the heat exchange unit in the stable cases (B in Fig. 1) to avoid total evaporation before the mist reached the test section. In the stable cases, the glycerine evaporator was installed in the upper part of the wind-tunnel section $(5 \times 5 \mathrm{~m})$ because the mist from the upper part of the underground section of the tunnel came around the wind-tunnel corners and filled the lower part of the test section on the upper floor. The bias behaviour was not a concern in the neutral and unstable cases because the glycerine mist went through the fan and a damper that mixed the air across the wind-tunnel section before it entered the heat exchange unit. Because the amount of glycerine required to fill the wind tunnel was fairly large due mainly to deposition on the large surface area especially in the heat exchangers (parts 23 and 24 in Fig. 1) and partly to the relatively low sensitivity of our LDA system, the mesh 
screens at the test-section entrance were covered with streaks of descending condensed glycerine after three consecutive vertical profile measurements. To prevent additional turbulence generation and non-uniformity in the airflow, the glycerine deposited in the wind tunnel was evaporated by heating the recirculating air to $90^{\circ} \mathrm{C}$ and was discharged through a ventilation pump for several hours after two vertical profile measurements. Because of this procedure, only two vertical profile measurements were possible per week.

Temperature was measured using a cold-wire probe (55P01, DISA, Denmark) consisting of a platinum-plated tungsten wire with a diameter of $5 \mu \mathrm{m}$ and a length of $3 \mathrm{~mm}$. The wire was placed parallel to the wind about $5 \mathrm{~mm}$ downwind of the cross volume of the LDA laser beams. The electric resistance of the wire was calibrated offline against the reading of a quartz thermometer (DMT-624, Tokyo Denpa Co., Ltd., Japan) at various values of the mainstream temperature. The sampling frequency was fixed at $200 \mathrm{~Hz}$. The mean, perturbation, and standard deviation of temperature are denoted by $T, \theta$, and $\sigma_{\theta}$, respectively.

The turbulent vertical heat flux $\overline{w \theta}$ was calculated by taking into account the separation $\Delta l$ of $5 \mathrm{~mm}$ between the cross volume of the LDA laser beams and the cold-wire probe. Based on the frozen turbulence hypothesis, $\overline{w \theta}$ was defined as the maximum value of $\overline{w(t) \theta(t+\tau)}$ as the delay $\tau$ was varied by small steps. The delay $\tau$ giving the maximum of $\overline{w(t) \theta(t+\tau)}$ was found to be approximately the same as $\Delta l / U$.

For the diffusion experiments, ethane diluted by nitrogen was emitted from a hole $13 \mathrm{~mm}$ in diameter that was located on the floor of the wind tunnel $12.3 \mathrm{~m}$ downwind of the test-section entrance and $0.975 \mathrm{~m}$ downwind of the upwind edge of the urban canopy model. The origin of $x$ was defined as the centre of this hole. The hole was filled with glass beads and then covered with a stainless steel mesh to prevent interference with the airflow above it. The glass beads also enhanced heat exchange between the ethane and the floor panel. The emission flow rate $Q_{\mathrm{v}}$ of the diluted ethane was $3.33 \times 10^{-6} \mathrm{~m}^{3} \mathrm{~s}^{-1}\left(0.2 \mathrm{~L} \mathrm{~min}^{-1}\right)$, equivalent to a vertical 
velocity of $0.025 \mathrm{~m} \mathrm{~s}^{-1}$ at the exit of the hole. The dilution ratio $r$ and the emission rate $Q=r Q_{\mathrm{v}}$ of ethane were chosen to keep the concentration within the dynamic range of the hydrocarbon detector.

The concentration of ethane in the region downwind from the source was measured by drawing air through a sampling rake to a flame ionization detector (FID, Kimoto Electric Co., Ltd. in Japan) calibrated using standard gas. The dashed lines in Fig. 2 indicate the $x$ positions where the $y z$-section of the concentration field was measured. At each rake position, measurement was continued until the accumulated average became nearly constant. The sampling rate was $1 \mathrm{~Hz}$, and the typical measurement time was $5 \mathrm{~min}$. More details of the concentration measurement technique are given in KY.

\section{Velocity and temperature field}

\subsection{Mean quantities}

Figure 4 shows the vertical profiles of $U$ and $T$. Inside the canopy $(z<H), U$ was approximately zero for all the conditions, the indication being that the measurement point was in the wake region of a block with very weak mean flow. The value of $U$ increased rapidly near the canopy top, and then followed a logarithmiclike relation up to approximately the middle of the boundary layer. The boundarylayer thickness $\delta$, defined as the height where $U$ reached about $98 \%$ of the ambient value of $U_{\infty}$ listed in Table 1 , was about $0.6-0.7 \mathrm{~m}$ in the stable and neutral cases and $0.8-0.9 \mathrm{~m}$ in the unstable cases. Because determination of $\delta$ was not a major concern in our study and also because of the limitation of duration of the measurement mentioned previously, the measurement interval at large $z$ was a rather coarse $0.1 \mathrm{~m}$ and the resolution of $\delta$ was low.

The mean temperature $T$ was normalized as $\left(T-T_{\mathrm{f}}\right) / \Delta T$, where $\Delta T=25^{\circ} \mathrm{C}$ was the temperature difference between the wind-tunnel floor and the inlet air. The temperature changed rapidly near the canopy top, and then followed a logarithmic- 
like relation. Inside the canopy, thermal stratification existed just as it did above the canopy.

We do not present further analysis on the profiles of $U$ and $T$ particularly in the region where they appeared to exhibit logarithmic-like relations because our measurements were limited to a single horizontal point in and above modelled urban canopy where considerable horizontal inhomogeneity has been known to exist (Cheng and Castro 2002).

\subsection{Turbulent fluctuations and fluxes}

Figure 5 shows the vertical profiles of the standard deviations (velocity fluctuations) $\sigma_{u}, \sigma_{v}$, and $\sigma_{w}$. The velocity fluctuations were scaled by $U_{\infty}$ rather than by the more physically relevant friction velocity $u_{*}$ because determination of $u_{*}$ could have large uncertainties. We clearly observed the velocity fluctuations increase as the stratification changed from stable to neutral to unstable.

Figure 6 shows the vertical profiles of $\sigma_{\theta}$, the Reynolds stress $\overline{u w}$, and the turbulent heat flux $\overline{w \theta}$. As was the case with velocity fluctuations, $\sigma_{\theta}$ was larger for unstable stratification than for stable stratification.

The Reynolds stress $\overline{u w}$ was small inside the canopy, but became abruptly very negative at the canopy top. Above the canopy, there was a constant-stress layer where the magnitude of $\overline{u w}$ was approximately constant. The magnitude of $\overline{u w}$ in the constant-stress layer became larger as the stratification changed from stable to neutral to unstable and also as the canopy height $H$ became larger. The thickness of the constant-stress layer became larger as the stratification changed from stable to neutral to unstable, but the top height of the layer appeared to be unaffected by the change in $H$.

The heat flux $\overline{w \theta}$ was negative, zero, and positive for stable, neutral, and unstable stratification, respectively, with some exceptions inside the canopy. We could not determine if the exceptions were evidence of genuine tendencies or were merely experimental errors. The magnitude of $\overline{w \theta}$ was relatively small in- 
$(\mathrm{a}-1)$

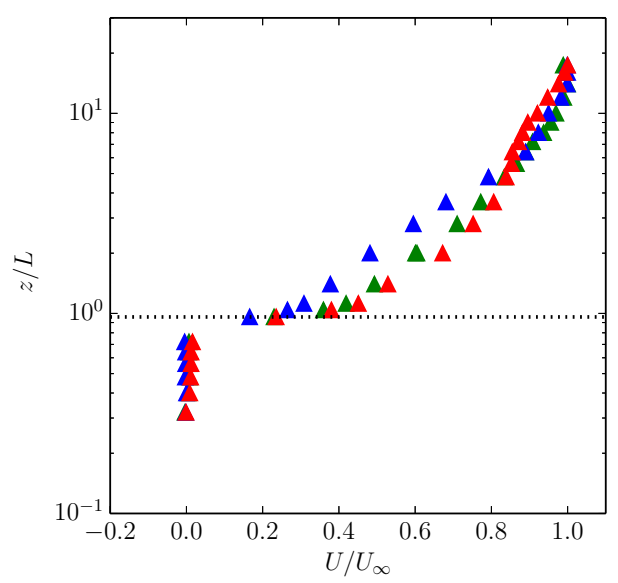

(b-1)

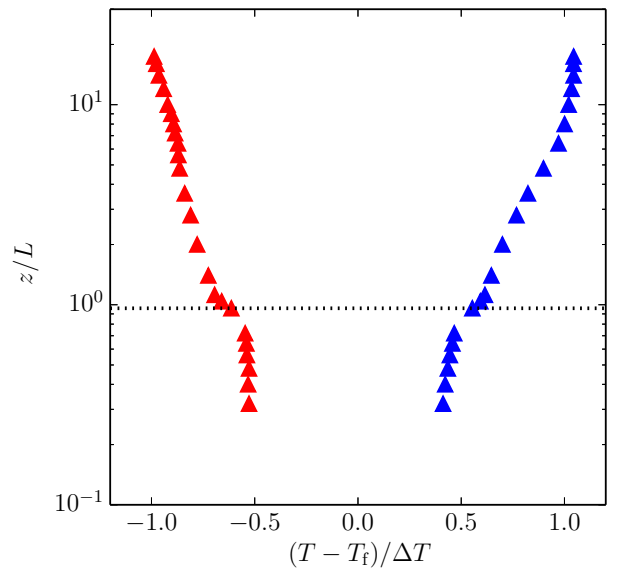

$(\mathrm{a}-2)$

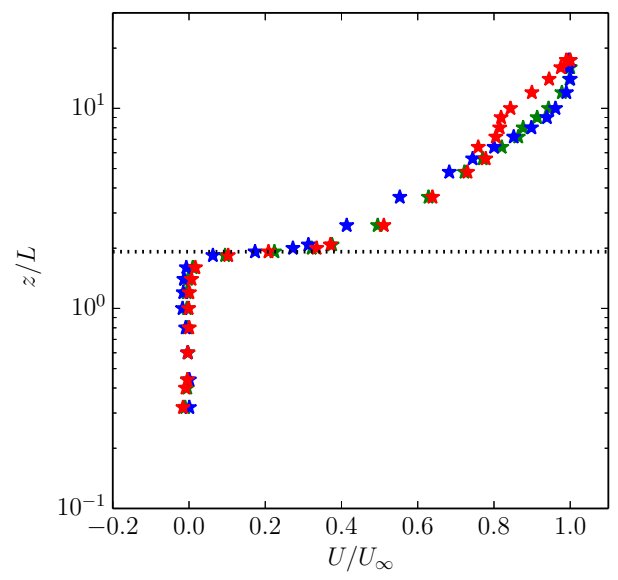

(b-2)

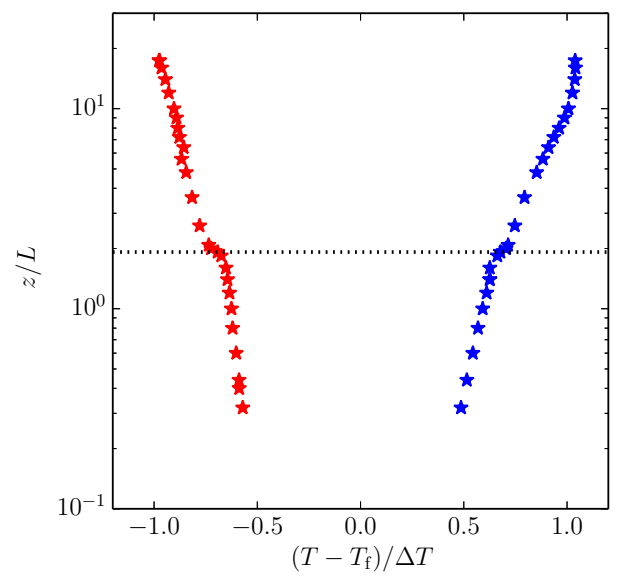

Figure 4: Vertical profiles of (a) the mean streamwise velocity $U$ and (b) the mean temperature $T$. The sub-labels 1 and 2 denote canopy heights $H=48 \mathrm{~mm}$ and $H=96 \mathrm{~mm}$, respectively. The blue, green, and red markers indicate stable, neutral, and unstable stratification, respectively. The horizontal dotted lines indicate the canopy top $z=H$. 
$(\mathrm{a}-1)$

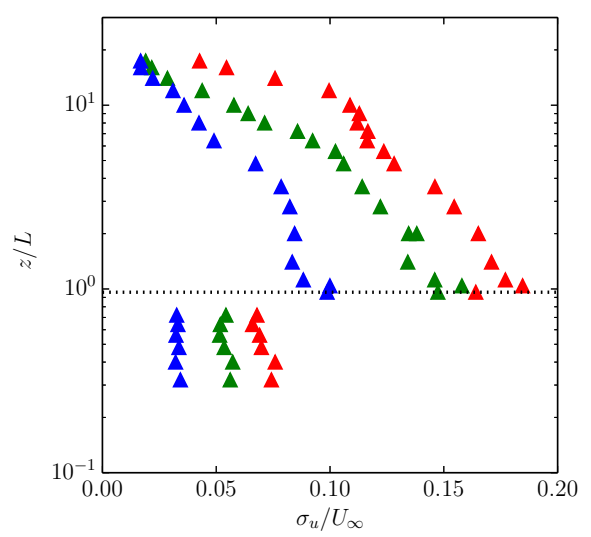

(b-1)

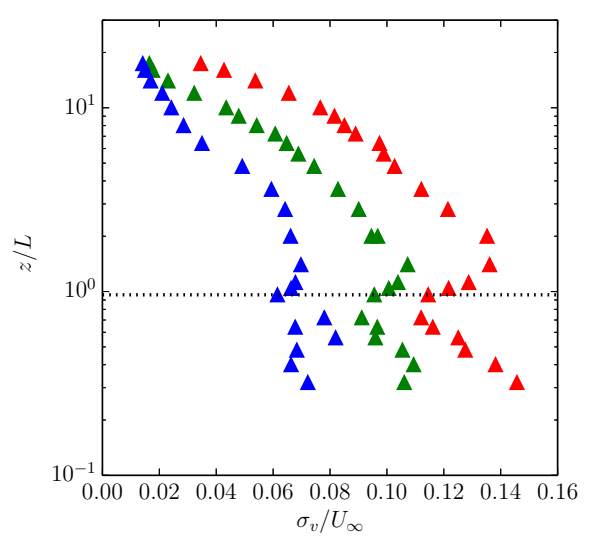

(c-1)

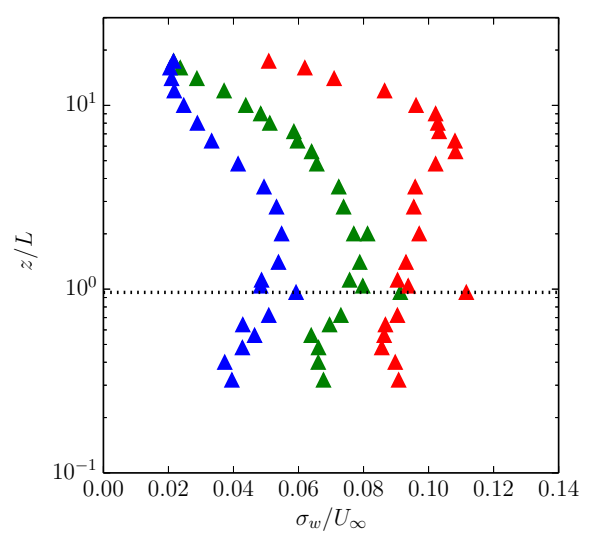

$(\mathrm{a}-2)$

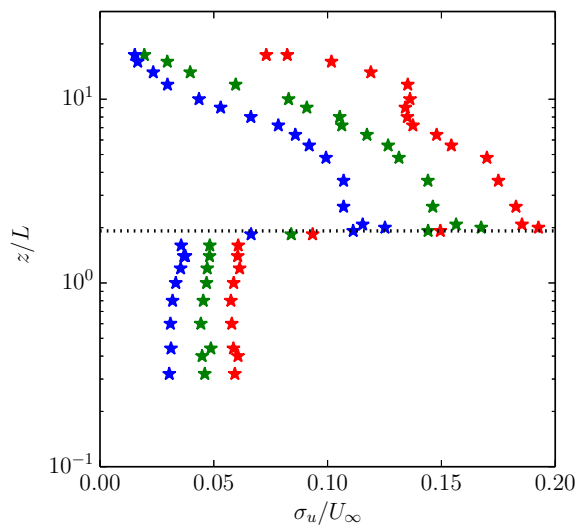

$(b-2)$

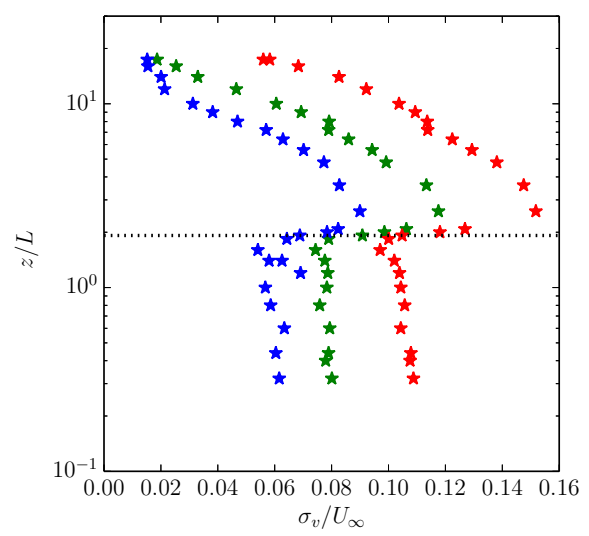

(c-2)

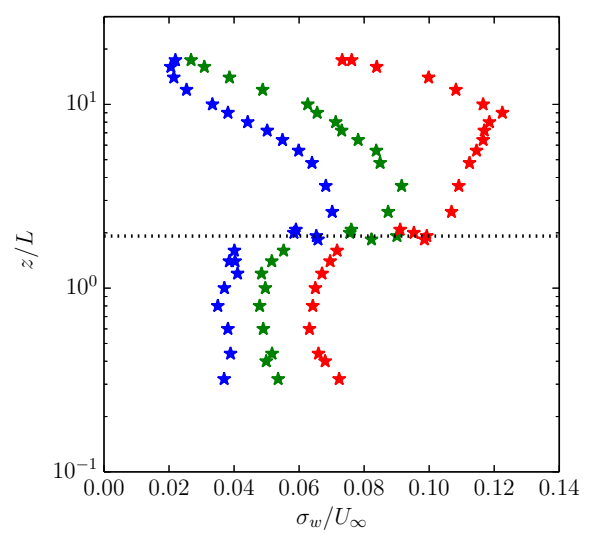

Figure 5: Vertical profiles of standard deviations of velocity components: (a) $\sigma_{u}$, (b) $\sigma_{v}$, and (c) $\sigma_{w}$. Columns 1 and 2 denote canopy heights $H$ of $48 \mathrm{~mm}$ and $96 \mathrm{~mm}$, respectively. The blue, green, and red markers indicate stable, neutral, and unstable stratification, respectively. The horizontal dotted lines indicate the canopy top $z=H$. 
(a-1)

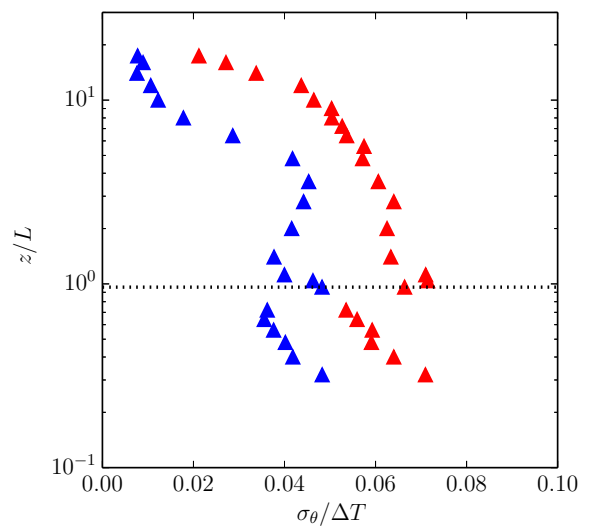

(b-1)

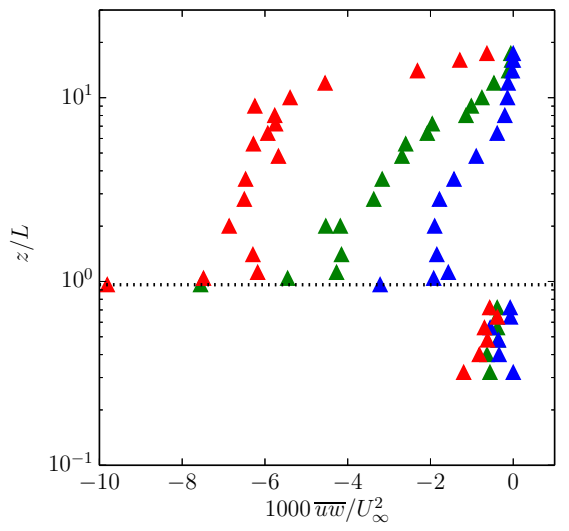

$(\mathrm{c}-1)$

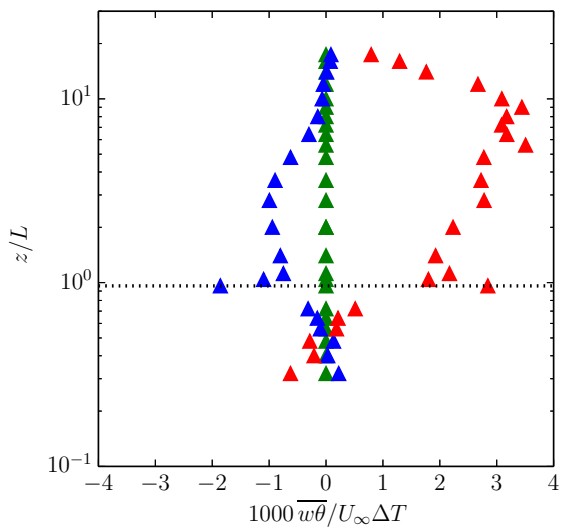

$(\mathrm{a}-2)$

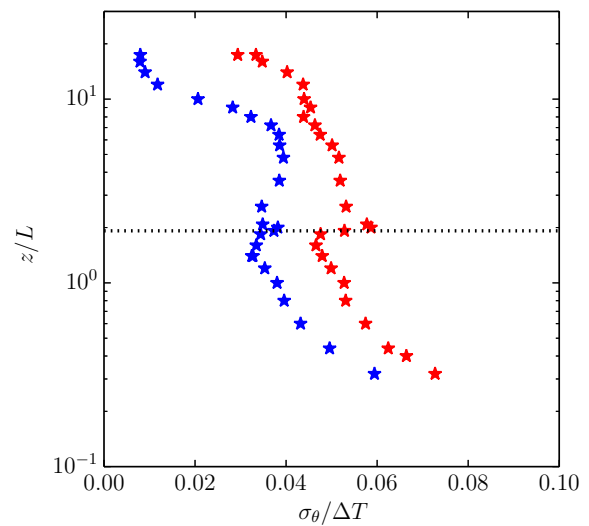

(b-2)

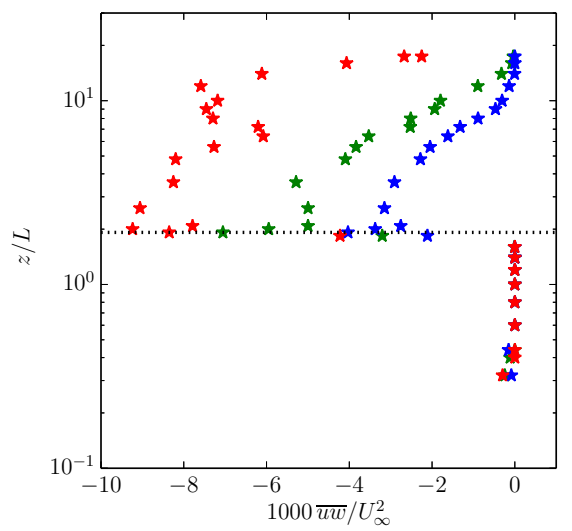

(c-2)

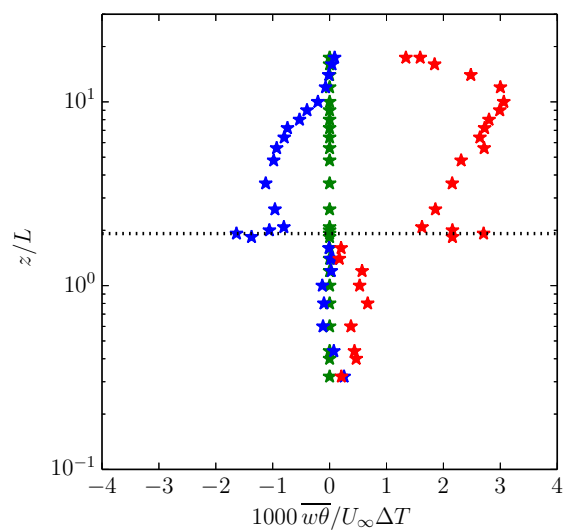

Figure 6: Vertical profiles of (a) standard deviation $\sigma_{\theta}$ of temperature, (b) momentum flux $\overline{u w}$, and (c) heat flux $\overline{w \theta}$. Columns 1 and 2 denote canopy heights $H$ of $48 \mathrm{~mm}$ and $96 \mathrm{~mm}$, respectively. The blue, green, and red markers indicate stable, neutral, and unstable stratification, respectively. 
side the canopy for both stable and unstable cases. Because heat flux must be continuous across the canopy top, there should be locations with relatively large $\overline{w \theta}$ inside the canopy so that the horizontally averaged heat flux matches at the canopy top. For the stable stratification, $\overline{w \theta}$ was approximately constant in the constant-stress layer, but for unstable stratification, $\overline{w \theta}$ increased up to $z / L \approx 10$ and decreased rapidly above that height. For experiments without spires and a fence at the test-section entrance, it is known that $\overline{w \theta}$ decreases monotonically with $z$ under unstable stratification conditions (e.g., Nagata et al. 2011). We considered the increase of $\overline{w \theta}$ for $z / L \lesssim 10$ under unstable stratification conditions to have been caused by the larger energetic eddies generated by the spires and fence, whereas for the stable stratification, large eddies were suppressed. Finally, there appeared to be negligible dependence of $\overline{w \theta}$ on $H$.

Fluctuations $\sigma_{u}, \sigma_{v}, \sigma_{w}$, and $\sigma_{\theta}$ were compared with field observation results in the surface atmospheric boundary layer summarized in Stull (1988). For the comparison, scaling parameters were defined as follows.

$$
\begin{aligned}
u_{*} & =\sqrt{-\overline{u w}_{\mathrm{CS}}}, \\
T_{*} & =-\frac{\overline{w \theta}_{\mathrm{CS}}}{u_{*}}, \\
L_{\mathrm{OB}} & =-\frac{T u_{*}^{3}}{g \kappa w \bar{C}_{\mathrm{CS}}},
\end{aligned}
$$

where the subscript ' $\mathrm{CS}$ ' denotes the constant-stress layer and $L_{\mathrm{OВ}}$ is the Obukhov length. Tables 1 and 2 list the values of the scaling parameters and the scaled fluctuations. We observe that the values of $L_{\mathrm{OB}}$ are comparable or larger than $\delta$, indicating very weak thermal stratification.

In field observations, the Obukhov length $L_{\mathrm{OB}}$ was used in the following similarity relations for the convective boundary layer (Stull 1988).

$$
\begin{aligned}
& \sigma_{w} / u_{*}=1.9\left(-z / L_{\mathrm{OB}}\right)^{1 / 3}, \\
& \sigma_{\theta} / T_{*}=-0.95\left(-z / L_{\mathrm{OB}}\right)^{-1 / 3} .
\end{aligned}
$$


Table 2: Scaling quantities based on the flux measurements, and scaled variances in the constantstress layer. The values of $z$ indicate the measurement heights considered representative of the constant-stress layer. In the case of stable stratification and $H=96 \mathrm{~mm}$, the values were averages at $z=130 \mathrm{~mm}$ and $z=180 \mathrm{~mm}$. The horizontal wind speed fluctuation $\sigma_{h}$ was defined by $\sigma_{h}=\left(\sigma_{u}^{2}+\sigma_{v}^{2}\right)^{1 / 2}$. Columns 'Stull' represent values of the similarity relations in the surface atmospheric boundary layer summarized in Stull (1988), recapitulated here as Eqs. 5 and 6.

\begin{tabular}{lccccccccccc}
\hline stability & \multicolumn{3}{c}{ Stable } & \multicolumn{3}{c}{ Neutral } & \multicolumn{5}{c}{ Unstable } \\
\hline$H(\mathrm{~mm})$ & 48 & 96 & Stull & 48 & 96 & Stull & 48 & Stul & 96 & Stull \\
$L_{\text {ов }}$ & 0.7 & 1.4 & & & & & -1.4 & -2.7 & \\
$z(\mathrm{~mm})$ & 100 & $130 / 180$ & & 100 & 180 & & 140 & 180 & \\
$\sigma_{u} / u_{*}$ & 1.93 & 1.95 & 2.4 & 2.06 & 2.02 & 2.5 & 1.91 & 1.92 & \\
$\sigma_{v} / u_{*}$ & 1.51 & 1.58 & 1.6 a) & 1.45 & 1.59 & $1.7-2.5$ & 1.50 & & 1.62 & \\
$\sigma_{h} / u_{*}$ & 2.45 & 2.51 & 2.9 & 2.52 & 2.57 & 2.9 & & & & \\
$\sigma_{w} / u_{*}$ & 1.26 & 1.26 & 1.6 & 1.18 & 1.28 & $1.0-1.6$ & 1.18 & 0.8 & 1.20 & 0.9 \\
$\sigma_{\theta} / T_{*}$ & 1.92 & 1.95 & 2.0 & - & - & - & -1.86 & -2.0 & -2.20 & -2.3 \\
\hline
\end{tabular}

a) calculated as $\sqrt{\sigma_{h}^{2}-\sigma_{u}^{2}} / u_{*}$ 
As shown in Table 2, most of the fluctuations in our experiments were smaller than those obtained by Eqs. 5 and 6 except for $\sigma_{w}$ for the unstable cases which were higher, and $\sigma_{w}$ for the neutral cases for which the experimental values were within the wide range of the field results.

For the unstable cases, convective velocity scale $w_{*}$ defined by

$$
w_{*}=\left(\frac{g}{T} \bar{w}_{\mathrm{CS}} \delta\right)^{1 / 3},
$$

may be of interest. Assuming $\delta=0.85 \mathrm{~m}$, we found $w_{*} / u_{*}=1.14$ and 0.92 for $H=48 \mathrm{~mm}$ and $H=96 \mathrm{~mm}$, respectively. These values are considerably smaller than the threshold 2.9 (Fedorovich 2004) for the distinction between shear and shear-free convection. Therefore, the established unstable stratification was confirmed again to be very weak.

\subsection{Integral length scales}

Integral length scales represent the sizes of turbulent eddies. Under Taylor's frozen turbulence hypothesis, an Eulerian integral length scale $L_{i}^{x}$ is defined by

$$
L_{i}^{x}=U(z) T_{i}^{x}=U(z) \int_{0}^{\infty} \rho_{i}(z, \tau) d \tau,
$$

where the subscript $i$ denotes the velocity component $u, v$, or $w$, where $T_{i}^{x}$ is the Eulerian integral time scale, and where the auto-correlation $\rho_{i}(z, \tau)$ is given by

$$
\rho_{i}(z, \tau)=\frac{\overline{u_{i}(z, t+\tau) u_{i}(z, t)}}{\overline{u_{i}^{2}}},
$$

where $u_{i}$ is either $u, v$ or $w$. In the following, we omit $z$ and consider a partial integral

$$
I_{i}(\tau)=\int_{0}^{\tau} \rho_{i}\left(\tau^{\prime}\right) d \tau^{\prime} .
$$

We note that Taylor's hypothesis has been shown to fail for free-shear flows in many experiments (Pope 2000), and the length scale (8) should be distinguished from the proper one defined by the spatial correlation. 
Ideally, $T_{i}^{x}$ should be obtained as the limiting value of $I_{i}(\tau)$ as $\tau$ becomes large. However, the measured $I_{i}(\tau)$ did not always behave as a function approaching a constant value for two reasons: (1) the uncertainty of $\rho_{i}(\tau)$ at large $\tau$ was large (equivalent to the uncertainty of the velocity spectrum at low frequencies), the result being irregular variations of $I_{i}(\tau)$ at large $\tau$, and (2) $\rho_{i}(\tau)$ had a distinct negative lobe for $i=v$ and $w$ at $z$ sufficiently elevated from the top of the roughness elements, the result being a decrease of $I_{i}(\tau)$ after a maximum.

To determine $T_{i}^{x}$ unambiguously, we adopted the following procedure. If $\rho_{i}(\tau)$ had a distinct negative lobe (determined subjectively from the plot of $I_{i}(\tau)$ ), $T_{i}^{x}$ was defined at the first zero of $\rho_{i}(\tau)$, i.e., at the maximum of $I_{i}(\tau)$. Otherwise, $T_{i}^{x}$ was calculated as the average of $I_{i}(\tau)$ in an appropriate interval. This interval was determined as follows. First, an initial guess $T_{0}$ of $T_{i}^{x}$ was defined as (see Pope 2000)

$$
T_{0}=S_{i}(0) / 4 \overline{u_{i}^{2}},
$$

where $S_{i}(0)$ is the value at $f=0$ of the one-sided power spectrum $S_{i}(f)$ of velocity fluctuation calculated by the maximum entropy method. Then, assuming an exponentially decaying form $\rho_{i}(\tau)=\exp \left(-\tau / T_{0}\right)$, an averaging interval $\left[\tau_{0}, \tau_{1}\right]$ was set such that $\rho_{i}\left(\tau_{0}\right)=0.01$ and $\rho_{i}\left(\tau_{1}\right)=0.001$ where 0.01 and 0.001 could be any other set of sufficiently small numbers. Finally, the time scale $T_{i}^{x}$ was calculated as the average of $I_{i}(\tau)$ in $\left[\tau_{0}, \tau_{1}\right]$, i.e.,

$$
T_{i}^{x}=\frac{1}{\tau_{1}-\tau_{0}} \int_{\tau_{0}}^{\tau_{1}} I(\tau) d \tau .
$$

If we regard $\rho_{i}(\tau)$ ill-bahaved when $\rho_{i}(\tau)$ did not have a distinct negative lobe and the difference between $T_{0}$ and $T_{i}^{x}$ was larger than 10\%, ill-bahaved $\rho_{i}(\tau)$ often occurred in $z / H \gtrsim 2$ for the neutral and unstable cases and independent of $z / H$ for the stable cases.

We note that repeating the above procedure with the right-hand side of Eq. 12 as a new initial guess $T_{0}$ until the difference from $T_{0}$ becomes sufficiently small is 
theoretically faulty. For example, if we assume an auto-correlation

$$
\rho_{i}(\tau)=e^{-\tau / T_{0}}+\epsilon \xi(\tau),
$$

where $\xi(\tau)$ is a random function with a small amplitude $\epsilon$, then Eq. 12 becomes

$$
T_{i}^{x}=0.996 T_{0}+\epsilon \int_{2 T_{0} \ln 10}^{3 T_{0} \ln 10} \int_{0}^{\tau} \xi\left(\tau^{\prime}\right) d \tau^{\prime} d \tau .
$$

For a simple case of $\epsilon=0$, Eq. 14 becomes $T_{i}^{x}=0.996 T_{0}$, quite acceptable considering the experimental errors. However, repetitive procedure implies solving

$$
T_{i}^{x}=\frac{\epsilon}{0.004} \int_{2 T_{i}^{x} \ln 10}^{3 T_{i}^{x} \ln 10} \int_{0}^{\tau} \xi\left(\tau^{\prime}\right) d \tau^{\prime} d \tau,
$$

which is independent of the unperturbed auto-correlation $e^{-\tau / T_{0}}$. Hence, the repetitive procedure was not adopted.

Figure 7 shows the vertical profiles of $L_{u}^{x}, L_{v}^{x}$, and $L_{w}^{x}$. Co-plotted in Fig. 7 are the integral length scales for the neutral-stability experiment with $U_{0}=3.5 \mathrm{~m} \mathrm{~s}^{-1}$ and with the urban-canopy models replaced by the upwind roughness bars in KY: the black and cyan markers indicate experiments with and without, respectively, the spires and fence at the test-section entrance. We first note that, in the neutral cases, the maximum values of $L_{u}^{x}$ were much larger $\left(L_{u}^{x} \approx 1.1 \delta\right)$ than those $\left(L_{u}^{x} \approx\right.$ 0.3 $)$ reported in previous studies (e.g., Robins 1979; Shirakata et al. 2002). As is apparent from the difference between the blue and black markers in Fig. 7, the large $L_{u}^{x}$ maxima were caused by the spires and fence. However, because the previous studies reporting $L_{u}^{x} \approx 0.3 \delta$ also installed spires, the large $L_{u}^{x}$ of our results can be attributed to the particular geometry of our spires, which rather excessively generated large energetic eddies of a size comparable to the boundarylayer height. In a preliminary experiment with smoke visualization, such large eddies were actually observed behind the spires. Although the spire shape and configuration were similar to those employed in Counihan (1969), the large height $(0.9 \mathrm{~m})$ compared to $\delta=0.7-0.8 \mathrm{~m}$ at the measurement location or geometrical errors due to the polystyrene body and the steel angle bars (see Fig. 3) could have 
$(\mathrm{a}-1)$

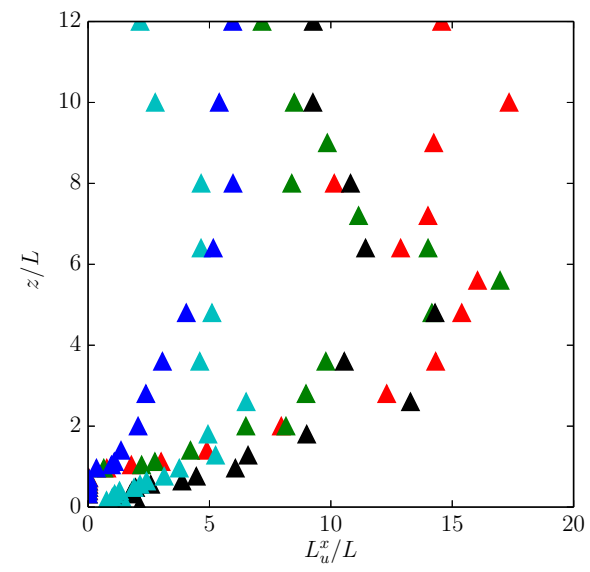

(b-1)

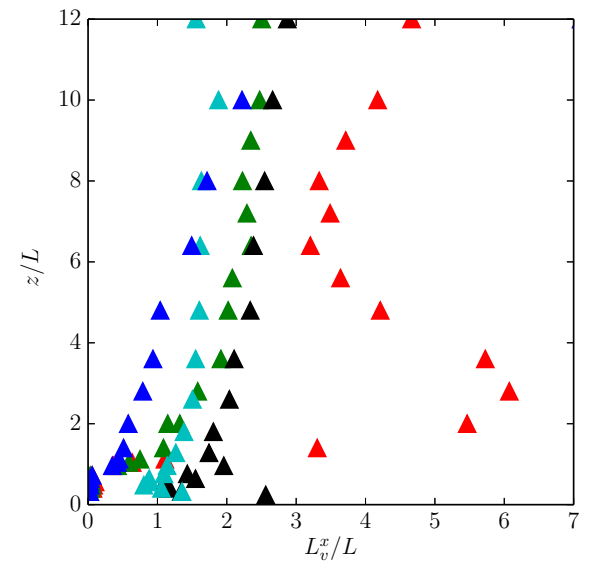

(c-1)

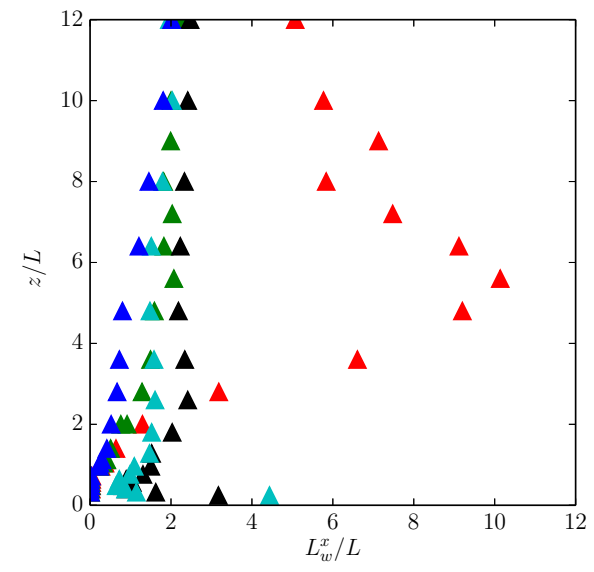

$(\mathrm{a}-2)$

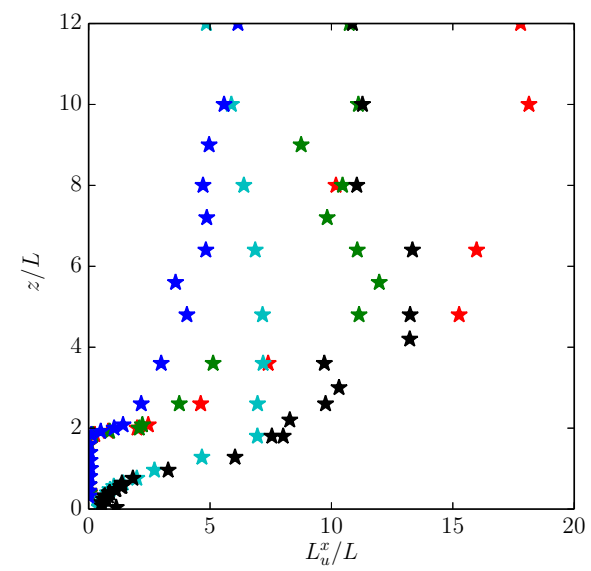

(b-2)

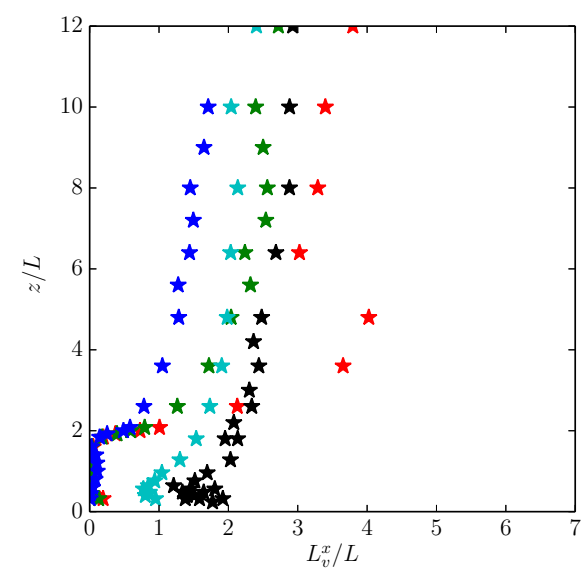

(c-2)

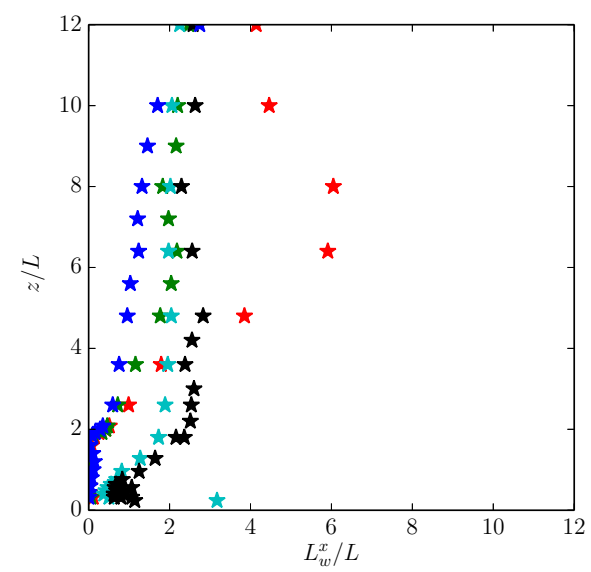

Figure 7: Vertical profiles of integral length scales: (a) $L_{u}^{x}$, (b) $L_{v}^{x}$, and (c) $L_{w}^{x}$. Columns 1 and 2 denote canopy heights $H$ of $48 \mathrm{~mm}$ and $96 \mathrm{~mm}$, respectively. The blue, green, and red markers indicate stable, neutral, and unstable stratification, respectively. The black and cyan markers indicate the results in $\mathrm{KY}$ with and without, respectively, the spires and fence at the test-section entrance under roughness conditions where the upwind roughness bars (h2 for $H=48 \mathrm{~mm}$, and h4 for $H=96 \mathrm{~mm}$ ) extend into the region of urban-canopy models. 
caused the large eddies. It should be noted that, with a triangular spire rather than an elliptic-wedge spire as employed in this study, Counihan (1969) found a conspicuous maximum of $\sigma_{u}$ around the middle height of the boundary layer.

The integral length scales $L_{i}^{x}$ increased as stratification changed from stable to neutral to unstable. Above the canopy top, $L_{i}^{x}$ increased monotonically with $z$ for all the components of the stable cases and for the $v$ and $w$ components of the neutral cases, but $L_{i}^{x}$ had maxima for all the components of the unstable cases and for the $u$ component of the neutral cases although the scatter of the data was large. The large scatter when the $L_{u}^{x}$ values are large was probably due to the measurement duration, which was not sufficiently long to reflect the long correlation time of the eddies with large $L_{u}^{x}$.

Particularly noteworthy were the very large maxima of the $v$ and $w$ components for the unstable stratification case. The difference from the neutral cases for these components was much larger than was the case for the $u$ component, which was predominantly controlled by the vorticity generated by the spires and fence. The locations and magnitudes of the maxima represented the locations and sizes of the turbulent eddies that were generated by both mechanical and convective forcings. Also, the difference of $L_{i}^{x}(i=u, v)$ due to the canopy height $H$ was substantially large (large differences in the overall magnitude, and in locations and magnitudes of the maxima) for the unstable stratification conditions, whereas the differences for neutral and stable stratification were relatively small (small difference in the profile shape and the overall magnitude). Hence, the morphology of the turbulent eddies was sensitive to the canopy geometry when convective forcing contributed to their generation.

\section{Concentration Field}

We examined the turbulent diffusion of a passive scalar in the presence of the urban canopy. In KY, diffusion under neutral stratification was studied by focusing on the effect of surface roughness conditions upwind of the urban canopy 
models. It was found that the concentration field near the source ( $x$ less than a few multiples of $L$ ) was little affected by the upwind roughness condition if the upwind fetch (distance between the upwind edge of the urban-canopy model and the source at $x=0$ ) was sufficiently large, but the concentration further downwind was affected considerably. The upwind fetch needed to eliminate the effect of the upwind roughness condition was much larger than that required for the adjustment of the second-order moments of turbulent velocity fluctuations. The upwind roughness bars $\mathrm{h} 2$ and $\mathrm{h} 4$ for the $H=48 \mathrm{~mm}$ and $H=96 \mathrm{~mm}$ urbancanopy blocks, respectively, employed in this study were found in $\mathrm{KY}$ to require the shortest fetch among the various bar heights tested for velocity field adjustment for each type of block, but the effect of the upwind roughness should appear in the concentration field at $x$ greater than a few multiples of $L$. Hence, the results presented below should be viewed with this consideration in mind.

Figure 8 shows the concentration field in the $y z$-plane at $x=2 L$ and $18 L$. To draw the contours, the measured values were interpolated onto a regular grid by the natural-neighbour interpolation method implemented in the ngmath library (http://ngwww.ucar.edu/ngmath/) of the NCL/NCAR Graphics package (NCAR 2012). The labelled values are a normalized concentration $C_{\mathrm{n}}$ defined by

$$
C_{\mathrm{n}}=\frac{C U_{\infty} L^{2}}{Q},
$$

where $C$ is the measured raw concentration in the volume mixing ratio. The fact that the spread of the plume became larger with $H$ and as the stratification changed from stable to neutral to unstable was consistent with the change of the relevant velocity fluctuations $\sigma_{v}$ and $\sigma_{w}$ (see Fig. 5). The axis of the plume deviated to negative $y$ for stable stratification. The deviation was due to the asymmetry in the thermal and geometrical conditions of the large wind tunnel and occurred with smaller amplitude even for the neutral cases of KY. In preliminary experiments, the magnitude of the deviation was found to become larger for larger $\Delta T$. Because the deviations were always in the opposite directions for stable and unstable 

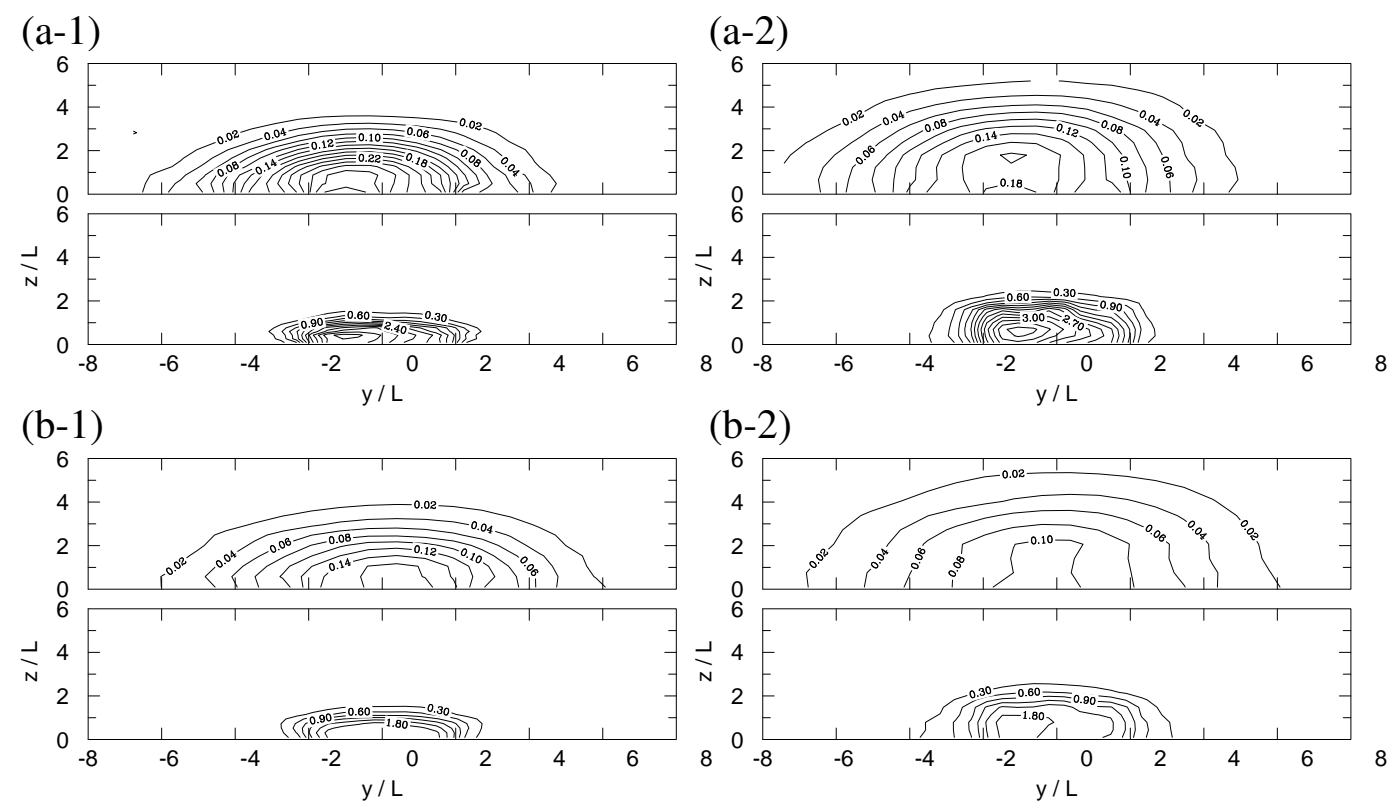

(b-2)
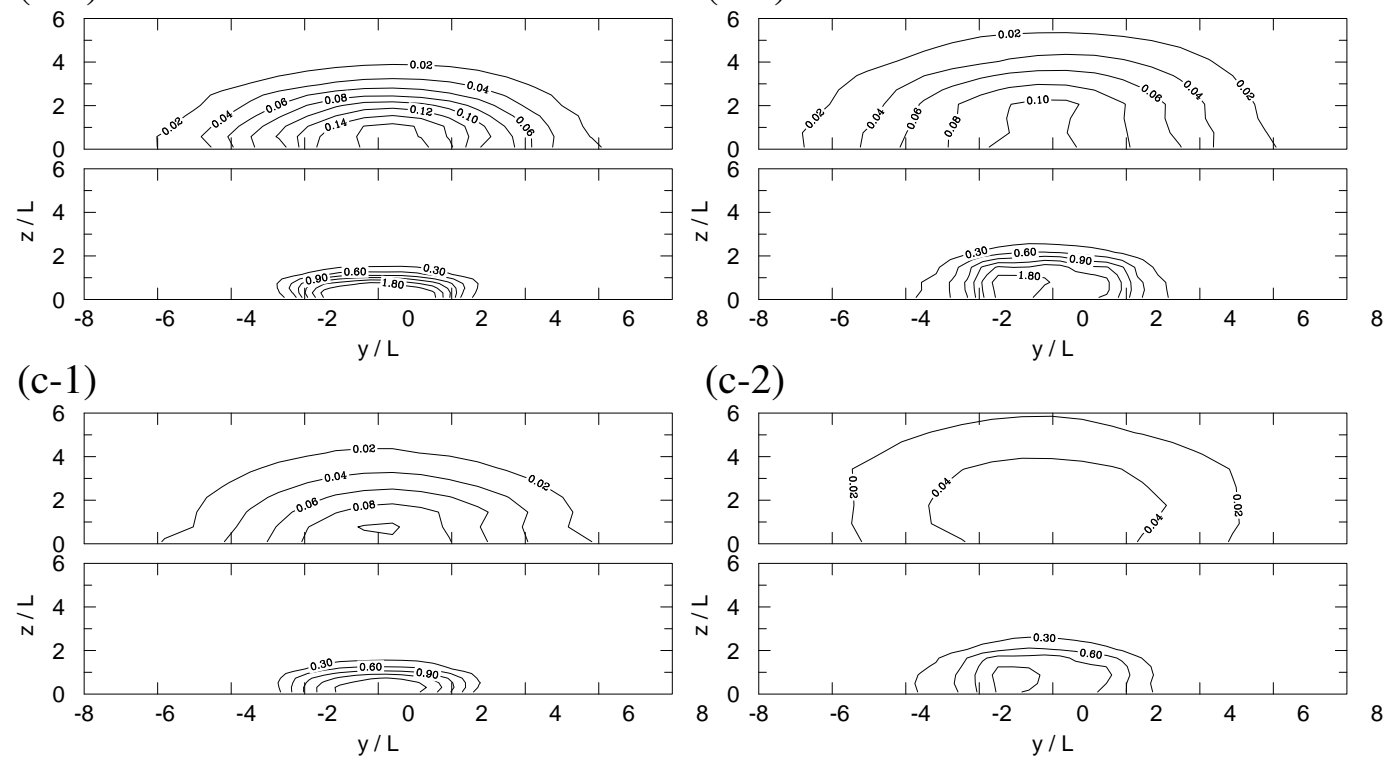

(c-2)

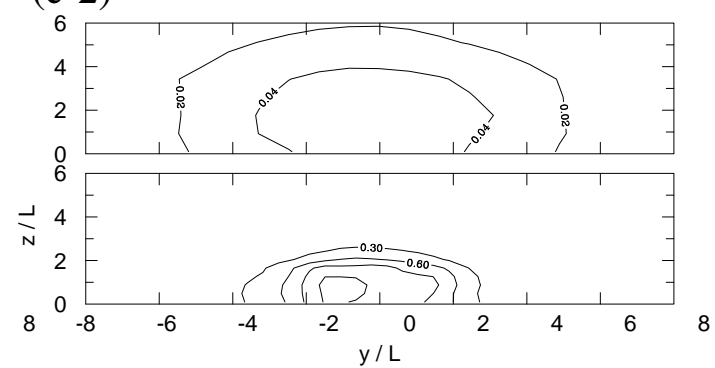

Figure 8: Contours of the normalized concentration $C_{\mathrm{n}}$ of ethane at two positions $(x)$ : lower panel $x=2 L$ (contour interval 0.3) and upper panel $x=18 L$ (contour interval 0.02). Columns 1 and 2 denote canopy heights $H$ of $48 \mathrm{~mm}$ and $96 \mathrm{~mm}$, respectively. The stratification is (a) stable, (b) neutral, and (c) unstable. 
cases with respect to the neutral cases, we suspect that secondary circulations that could not be removed completely by the installed internal walls were the cause of the deviations. This is one of the reasons why we restricted our experiments to relatively weak stratification.

Figure 9 shows the vertical profiles of $C_{\mathrm{n}}$ at the centerline $y=0$. At $x=2 L$, for all the conditions, $C_{\mathrm{n}}$ had a maximum at a little below the model half height. This in-canopy peak appeared to persist to $x=4 L, 4 L$, and $8 L$ for unstable, neutral, and stable conditions, respectively, for $H=48 \mathrm{~mm}$ (Fig. 9a), and to $x=2 L, 8 L$, and $12 L$ for unstable, neutral, and stable conditions, respectively, for $H=96 \mathrm{~mm}$ (Fig. 9b). The persistence of the peak for the stable stratification implies suppressed vertical mixing below the model height. Difference due to the canopy height could not be deduced confidently with the vertical resolution of our measurement.

The crosswind profiles of $C_{\mathrm{n}}$ at $z=0.1 L$ and $x=L$ or $2 L$ are shown in Fig. 10. The thick horizontal lines indicate the locations of the blocks immediately upwind of the measurement line. At $x=L, C_{\mathrm{n}}$ was almost uniform in $|y|<$ $1.5 \mathrm{~L}$ because the block immediately downwind of the source mixed the tracer gas efficiently. Asymmetry about $y=0$ was observed clearly under the stable stratification condition even in the near-source region. At $x=2 L$, channelling effect (e.g., Robins et al. 2002) could be observed; $C_{\mathrm{n}}$ was nearly uniform across an upwind opening and decayed stepwise across an upwind blockage.

The concentration field $C(y, z)$ in the $y z$-plane at each $x$ was fitted to a modified standard form (Hunt and Weber 1979):

$$
C=C_{0} \exp \left[-\frac{\left(y-y_{0}\right)^{2}}{2 \sigma_{y}^{2}}-\left(\frac{A z^{\prime}}{\sigma_{z}}\right)^{s}\right],
$$

where $C_{0}$ is the maximum concentration at the $x$ of concern, $\sigma_{y}(x)$ and $\sigma_{z}(x)$ are the $y$-and $z$-direction plume widths, respectively, as functions of $x$, and $y_{0}$ is the $y$ coordinate of the plume axis. The modified vertical coordinate $z^{\prime}$ was introduced 
(a) $x=2 L$

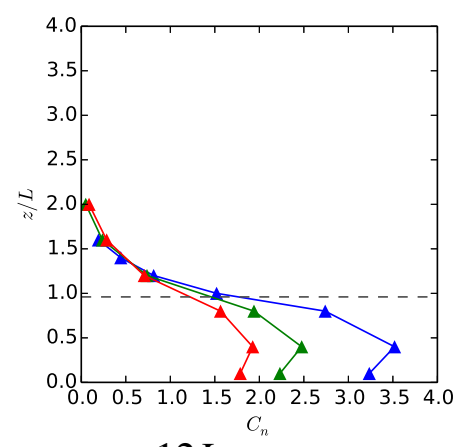

$x=12 L$

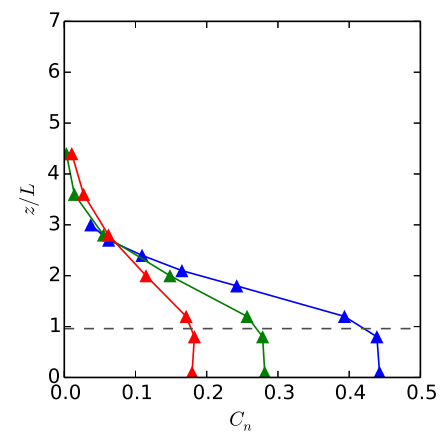

(b) $x=2 L$

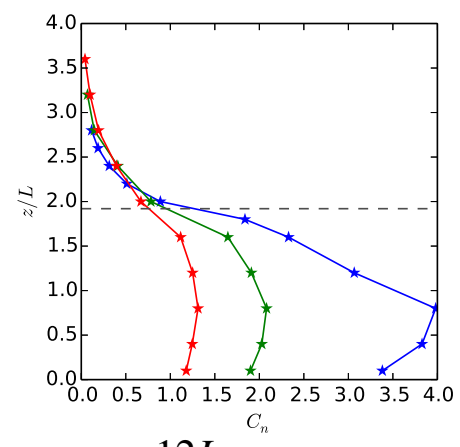

$x=12 L$

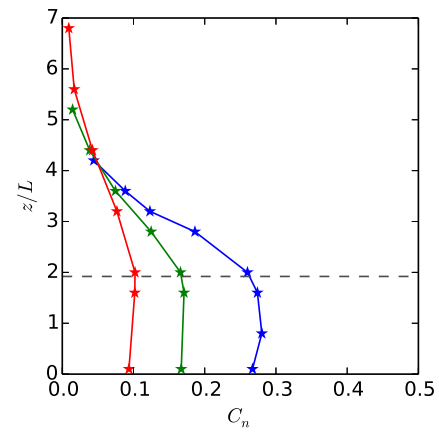

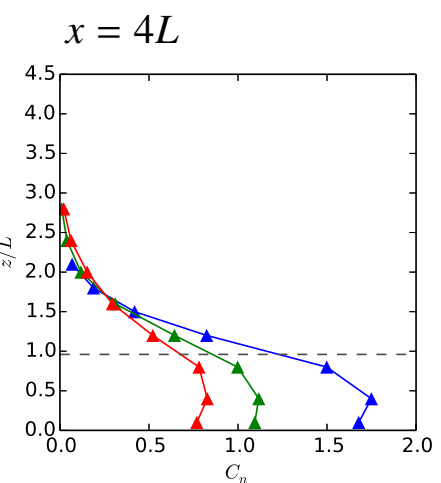
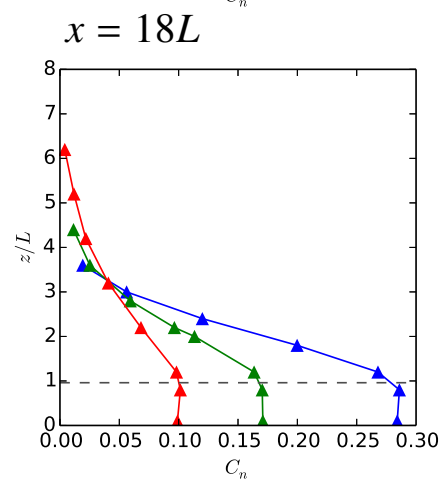

$$
x=4 L
$$

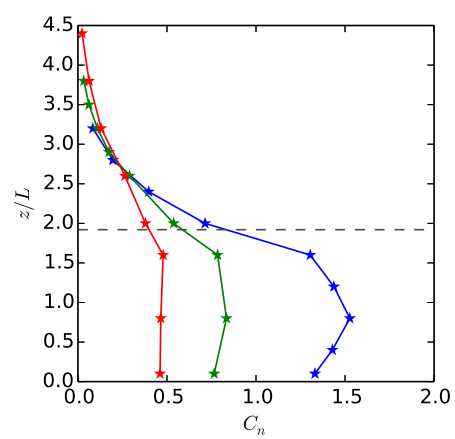

$$
x=18 L
$$

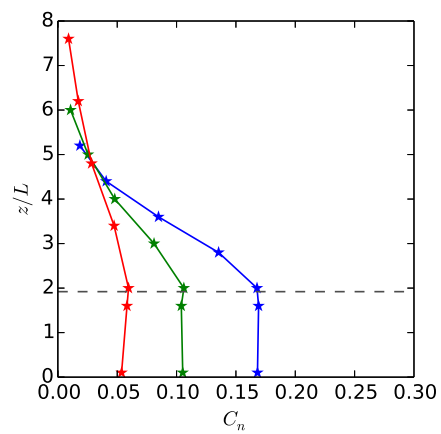

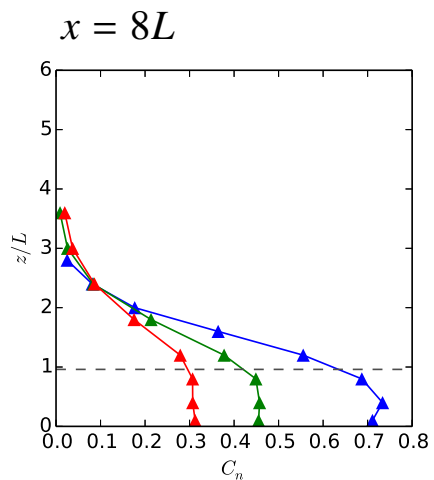

$x=24 L$

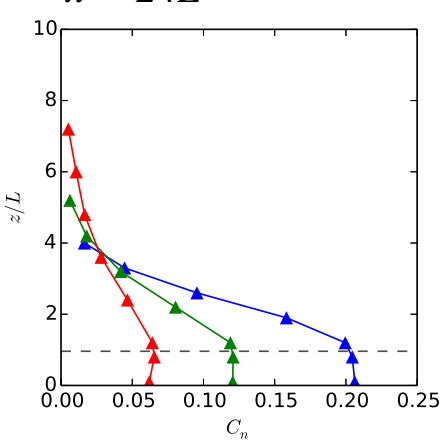

$x=8 L$

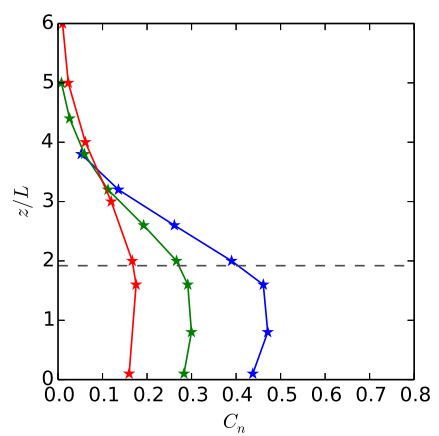

$$
x=24 L
$$

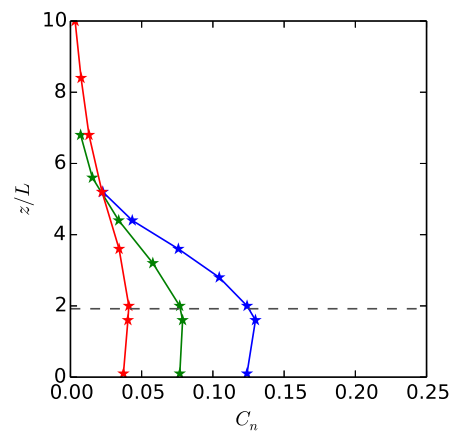

Figure 9: Vertical (z) profiles of the normalized concentration $C_{\mathrm{n}}$ for $H=48 \mathrm{~mm}$ (a) and $96 \mathrm{~mm}$ (b). The grey dashed lines indicate the canopy top. The blue, green, and red markers indicate stable, neutral, and unstable stratification, respectively. 
$(\mathrm{a}-1)$

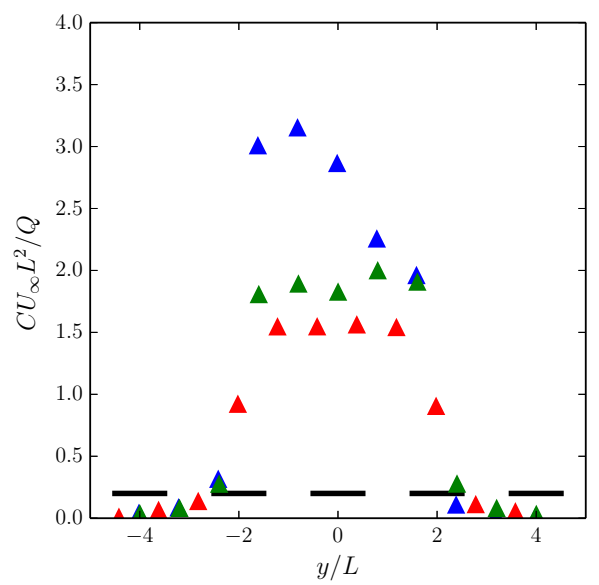

(b-1)

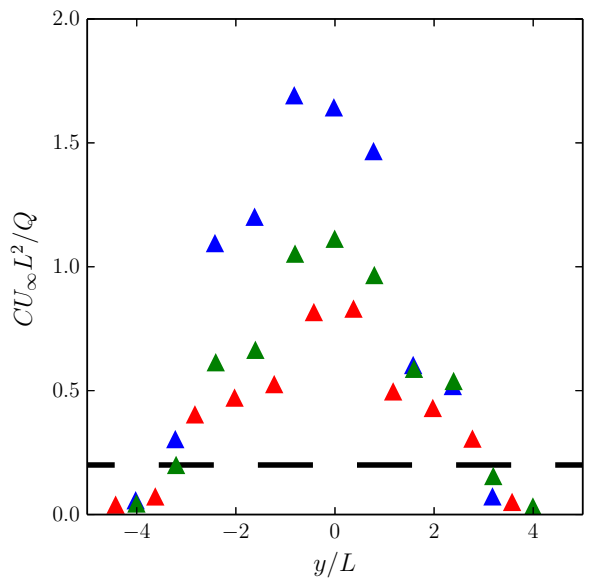

$(\mathrm{a}-2)$

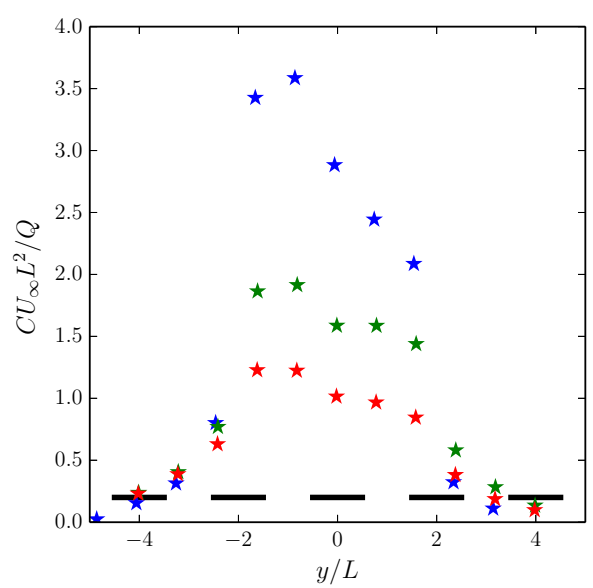

(b-2)

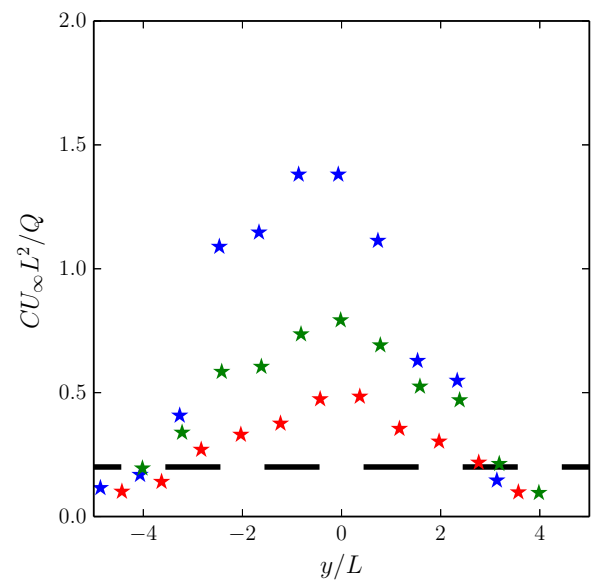

Figure 10: Crosswind (y) profiles of the normalized concentration $C_{\mathrm{n}}$ at $z=0.1 L$ and $x=L$ (a) or $x=2 L$ (b). The thick horizontal lines indicate the locations of the blocks immediately upwind of the measurement line. Columns 1 and 2 denote canopy heights $H$ of $48 \mathrm{~mm}$ and $96 \mathrm{~mm}$, respectively. The blue, green, and red markers indicate stable, neutral, and unstable stratification, respectively. 
here to account for the well-mixed canopy region

$$
z^{\prime}= \begin{cases}z-d & (z>d) \\ 0 & (z \leq d)\end{cases}
$$

where $d$ is a fitting parameter that is a result of both advection and diffusion inside the canopy, and is assumed to change with the downwind distance $x$. As mixing inside the canopy proceeds, $d$ tended to $H$ within experimental errors, which was reasonable considering the vigorous mixing in $z \leq H$. The coefficient $A$ is defined as

$$
A=[\Gamma(3 / s) / \Gamma(1 / s)]^{(1 / 2)},
$$

where $\Gamma$ represents the gamma function defined by

$$
\Gamma(s)=\int_{0}^{\infty} t^{s-1} e^{-t} \mathrm{~d} t .
$$

For reasons discussed in KY, we set $s=1.5$. Strictly speaking, the value of $s$ is a function of stability, but it was assumed constant because the parameter determination became less robust if $s$ was set as another free parameter. The parameters $C_{0}, \sigma_{y}, \sigma_{z}, y_{0}$, and $d$ were determined by the Nelder-Mead method. A large uncertainty in the determined parameters occurred for unstable stratification where the concentration field was skewed considerably as shown in Fig. 8. To reduce this uncertainty, the values of $d$ determined for the neutral cases were adopted for non-neutral cases. Note that the parameter values determined in this way were not that different from those determined with $d$ as an optimization parameter as to alter the qualitative features discussed below.

Figure 11 shows the downwind variation of $C_{0}$ for non-neutral cases. To provide a comparison with the values for neutral stratification, $C_{0}$ was normalized as

$$
\frac{C_{0} U_{\mathrm{H}} / Q}{C_{0, \text { neut }} U_{\mathrm{H}, \text { neut }} / Q_{\text {neut }}},
$$

where $U_{\mathrm{H}}$ was the wind speed at the canopy top $z=H$, and the subscript 'neut' denotes values for neutral stratification. For the shorter canopy with $H=48 \mathrm{~mm}$, 
(a)

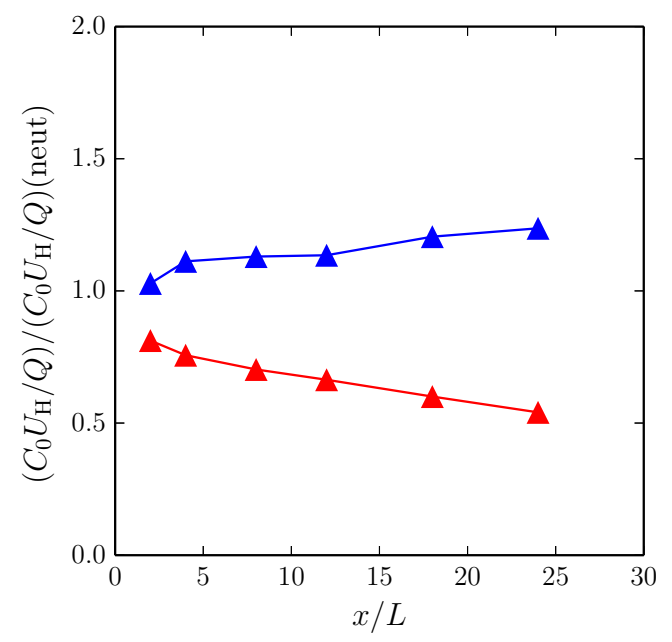

(b)

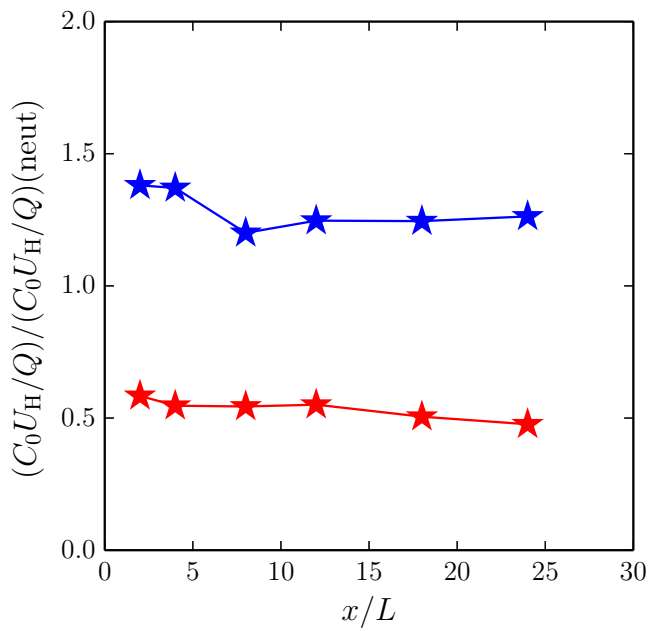

Figure 11: Downwind evolution of the maximum concentration $C_{0}$ in the $y z$-plane relative to the value in the corresponding neutral case: (a) $H=48 \mathrm{~mm}$, and (b) $H=96 \mathrm{~mm}$. The blue and red markers indicate stable and unstable stratification, respectively.

the difference from neutral cases increases monotonically with the downwind distance $x$. This behaviour is consistent with the intuitive picture that the relative importance of thermal stratification is small in the vicinity of the source where the eddies generated by the canopy blocks have a dominant influence on diffusion, but becomes larger downwind as the plume grows beyond the size of individual blocks. However, for the taller canopy with $H=96 \mathrm{~mm}$, the difference from the neutral cases did not depend much on the downwind distance. This result is contrary to intuition because mechanical mixing is considered to be more vigorous for the taller canopy. However, as shown in Fig. 5, turbulence intensity was actually smaller for the taller canopy due to more effective shear-sheltering. Also, the taller canopy blocks are expected to generate taller eddies that would be more susceptible to stratification.

Figure 12 shows the downwind growth behaviours of $\sigma_{y}, \sigma_{z}$, and $d$. As noted in the case of Fig. 8, the plume widths grew with $x$ more rapidly as the stratification changed from stable to neutral to unstable. At $x=2 L$, the measurement line immediately downwind of the source, the changes of $\sigma_{y}$ and $\sigma_{z}$ due to the strati- 
(a-1)

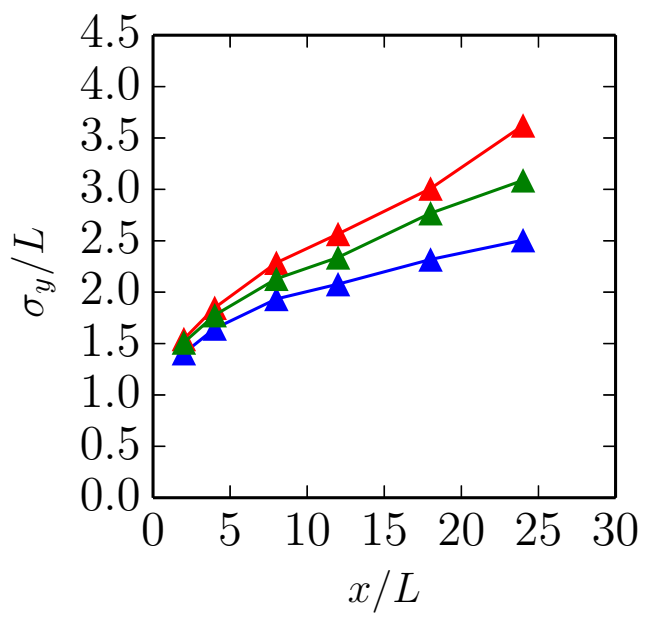

(b-1)

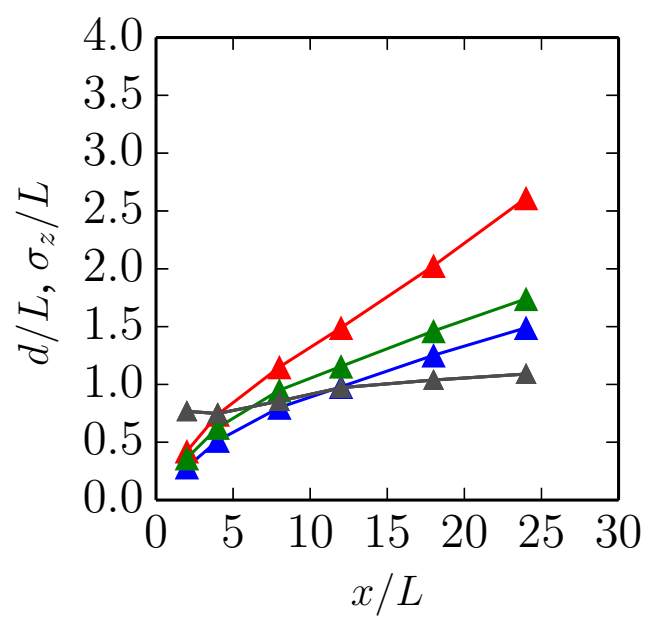

$(\mathrm{a}-2)$

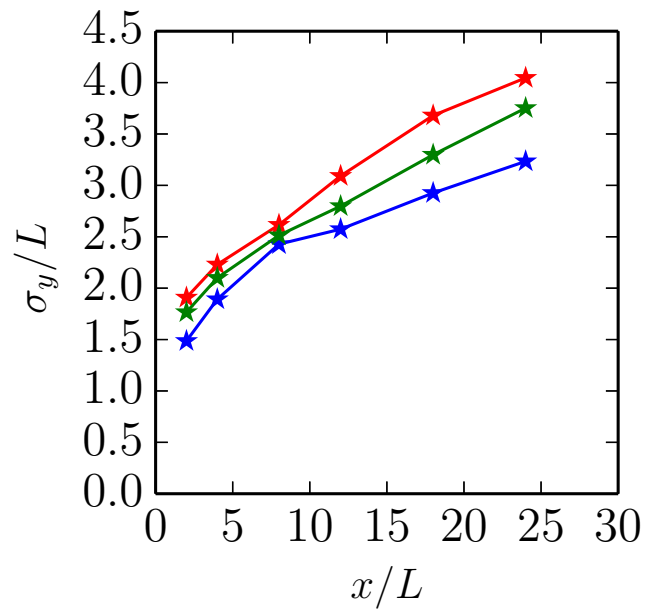

(b-2)

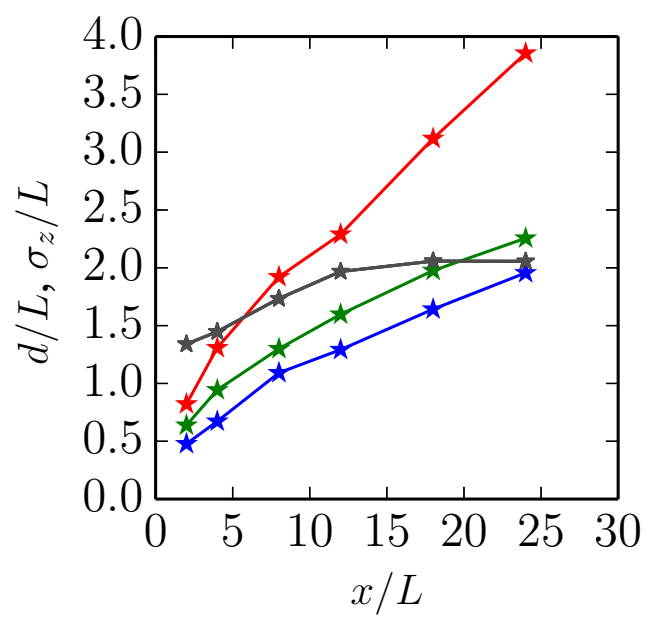

Figure 12: Downwind growth of the plume widths (a) $\sigma_{y}$ and (b) $\sigma_{z}$ and $d$ (b). Columns 1 and 2 denote canopy heights $H$ of $48 \mathrm{~mm}$ and $96 \mathrm{~mm}$, respectively. The grey markers in (b) represent $d / L$. The blue, green, and red markers indicate stable, neutral, and unstable stratification, respectively. 
Table 3: Briggs formulas for plume widths over urban roughness.

\begin{tabular}{ccc}
\hline Stability class & $\sigma_{y}(\mathrm{~m})$ & $\sigma_{z}(\mathrm{~m})$ \\
\hline C & $0.22 x(1+0.0004 x)^{-1 / 2}$ & $0.20 x$ \\
D & $0.16 x(1+0.0004 x)^{-1 / 2}$ & $0.14 x(1+0.0003 x)^{-1 / 2}$ \\
E-F & $0.11 x(1+0.0004 x)^{-1 / 2}$ & $0.08 x(1+0.0015 x)^{-1 / 2}$ \\
\hline
\end{tabular}

fication condition were small because vigorous turbulent mixing inside the urban canopy reduced the effect of thermal stratification. We observed that the changes of $\sigma_{y}$ and $\sigma_{z}$ at $x=2 L$ became larger for $H=96 \mathrm{~mm}$ than for $H=48 \mathrm{~mm}$, a result consistent with the explanation of the results in Fig. 11.

We observed that stable stratification affected mainly $\sigma_{y}$, but had smaller effect on $\sigma_{z}$. It is worthwhile to compare our results with the Briggs formulas (Briggs 1973) representing field experiments over urban roughness. Table 3 lists the formulas for the Pasquill-Gifford stability classes C (weakly unstable), D (neutral), and E-F (weakly to moderately stable). In these formulas, change in stratification from neutral to stable at relatively small $x$, where the power-law term can be regarded as unity, had more effect on $\sigma_{z}$ (43\% reduction) than on $\sigma_{y}(31 \%$ reduction). Similarly, for the change from neutral to unstable stratification, the change of $\sigma_{z}$ (43\% increase) was larger than the change of $\sigma_{y}$ (25\% increase). Because in an open space without considerable blockage, such as urban canopy, thermal stratification should affect primarily vertical motions, it is reasonable that the relative change in $\sigma_{z}$ was larger than the change in $\sigma_{y}$. In our experiments, the relative difference in the magnitudes of $\sigma_{w}$ between stable and neutral conditions (Fig. 5c) was comparable to the relative difference in the magnitudes of $\sigma_{v}$ (Fig. 5b). Also, the relative difference in the magnitudes of $L_{w}^{x}$ between stable and neutral conditions (Fig. 7c) was comparable to the relative difference in the magnitudes of $L_{v}^{x}$ (Fig. 7b). Therefore, our result that the growth rate of $\sigma_{z}$ for stable stratification was so close to that for neutral stratification cannot be explained readily by 
conventional knowledge. Note that, in preliminary experiments with the urbancanopy models replaced with the upwind roughness elements, we had observed a distinct difference in the growth rates of $\sigma_{z}$ between stable and neutral stratification conditions. Note that these preliminary experiments were conducted in the presence of considerable secondary circulation because the temperature difference $\Delta T$ was large. Whether our current result was due to experimental errors or was caused by some mechanism peculiar to stable stratification cannot be determined with the available experimental data. Further investigation will be necessary to elucidate the cause of this behaviour.

Additional aspect of the diffusion behaviour can be examined in terms of the vertical entrainment velocity $w_{\mathrm{e}}$ defined by

$$
w_{\mathrm{e}}=\frac{d}{d x}\left(\frac{Q}{\sqrt{2 \pi} \sigma_{y} C_{0}}\right) .
$$

By analysis of diffusion experiments (quasi-two-dimensional geometry using line sources) in three different wind tunnels under neutral stratification conditions with rough surfaces, Britter et al. (2003) found

$$
w_{\mathrm{e}}=\alpha_{\mathrm{E}} u_{*},
$$

where the entrainment coefficient $\alpha_{\mathrm{E}}$ was $0.65 \pm 0.05$. It should be noted that, in the analysed three wind-tunnel studies (Robins et al. 2001a; Havens et al. 2001; Snyder 2001), the zero-plane displacement was assumed zero because the roughness elements had negligible horizontal section area (small $\left.\lambda_{\mathrm{p}}\right)$. Britter et al. (2003) also deduced $\alpha_{\mathrm{E}}=0.63 \pm 0.05$ for the near-neutral conditions of the Prairie Grass field experiment. In our experiments with three-dimensional plumes, it was found that $1 / \sigma_{y} C_{0}$ changed linearly with $x$ in $x \geq 12 L$. Table 4 lists the values of $\alpha_{\mathrm{E}}$ obtained as the slope of $Q / \sqrt{2 \pi} \sigma_{y} C_{0}$ against $x$ in $x \geq 12 L$. For the stable and neutral stratification conditions, the values of $\alpha_{\mathrm{E}}$ were found to be smaller than those reported by Britter et al. (2003). The small difference in $\alpha_{\mathrm{E}}$ between stable and neutral conditions in contrast to the much larger value for the unstable 
Table 4: Vertical entrainment velocity

\begin{tabular}{lcccccc}
\hline stability & \multicolumn{2}{c}{ Stable } & \multicolumn{2}{c}{ Neutral } & \multicolumn{2}{c}{ Unstable } \\
\hline & & & & & & \\
$H(\mathrm{~mm})$ & 48 & 96 & 48 & 96 & 48 & 96 \\
$w_{\mathrm{e}}\left(\mathrm{m} \mathrm{s}^{-1}\right)$ & 0.044 & 0.055 & 0.061 & 0.071 & 0.115 & 0.159 \\
$w_{\mathrm{e}} / u_{*}$ & 0.49 & 0.46 & 0.47 & 0.45 & 0.75 & 0.90 \\
\hline
\end{tabular}

condition is an alternative expression of the diffusion behaviour discussed in the previous paragraph.

\section{Summary and discussions}

An urban geometry was simulated in a wind tunnel using staggered arrays of parallelepiped blocks. By controlling the temperature of the recirculating air and the test-section floor, weakly stable and unstable stratification conditions were established such that the Obukhov length was larger than the boundary-layer thickness. Vertical profiles of the velocity components and temperature were measured at a point in the lee of a block using laser-Doppler anemometry and a cold-wire thermometer. Despite the weak stratification, velocity and temperature fluctuations were found to be sensitive to stratification. Passive-scalar diffusion from the wind-tunnel floor was studied using ethane as a tracer gas. Even in the proximity of the source, where diffusion occurred mostly in the urban-canopy region, the spread of the tracer-gas plume was found to be highly dependent on the degree of stratification.

The important features of our study are: (i) stable, neutral, and unstable stratification was realized in the same urban-canopy geometry, (ii) the seeding particles for the LDA measurement were dispersed as uniformly as possible, and (iii) the tracer gas for the diffusion study was emitted from the wind-tunnel floor. The first aspect (i) has been realized in the past for a single-block (Yassin 2013) or hill geometry (Ohba et al. 2002), but not for an urban-canopy geometry. The reason is 
probably that laying hundreds of blocks on a bare wind-tunnel floor is a laborious task. We emphasize that we still used simpler roughness elements in the upwind region of the urban-canopy models. As mentioned in Section 4, insufficient adjustment of the velocity field across the roughness change could have non-negligible effects on the diffusion behavior. Numerical studies such as Boppana et al. (2014) would be a viable approach to this problem.

The second aspect (ii) is essential to make correct measurements. In preliminary experiments, we once introduced seeding particles from a perforated vertical pipe located a little downwind of the spires. When compared with the measurement data using this paper's method of seeding, the velocity fluctuation was considerably smaller because the narrow and non-uniform seeding could not capture the large-scale eddies. As for the third aspect (iii), we note that a similar experiment on a smooth surface would not be appropriate because the emission velocity would be comparable to or larger than the velocity of the airflow near the source. In our experiments, velocity mismatch near the source did exist, but vigorous mechanical mixing in the canopy region rapidly spreads the tracer gas and the velocity mismatch had a negligible effect as described in KY.

\section{Acknowledgements}

The authors appreciate valuable advice from Dr. K. Uehara in conducting the experiments, and the suggestion by Dr. Y. Sakai to fill the wind tunnel with the seeding particles. Unfortunately, the wind tunnel was demolished in 2014, 36 years after its construction in March 1978. We are grateful to all the people who have been engaged in the management of the wind tunnel and related research. This work was partially supported by the Joint Research Project on Formation Mechanism of Ozone, VOCs, and $\mathrm{PM}_{2.5}$ and Proposal of Countermeasure Scenarios funded by the Japanese agencies JST and JICA under the SATREPS scheme. 


\section{References}

\section{References}

Arya SPS (1975) Buoyancy effects in a horizontal flat-plate boundary layer. J. Fluid Mech. 68:321-343

Boppana VBL, Xie ZT, Castro IP (2014) Thermal stratification effects on flow over a generic urban canopy Boundary-Layer Meteorol 153: 141-162

Briggs GA (1973) Diffusion estimation for small emissions. In Atmospheric Turbulence and Diffusion Laboratory Report. NOAA/ATDL ATDL-106.

Britter RE, Hanna SR, Briggs GA, Robins A (2003) Short-range vertical dispersion from a ground level source in a turbulent boundary layer. Atmos Environ $37: 3885-3894$

Counihan J (1969) An improved method of simulating an atmospheric boundary layer in a wind tunnel. Atmos Environ 3:197-214

Cheng H, Castro IP (2002) Near wall flow over urban-like roughness. BoundaryLayer Meteorol 104:229-259

Fedorovich E (2004) Dispersion of passive tracer in the atmospheric convective boundary layer with wind shears: a review of laboratory and numerical model studies. Meteorol Atmos Phys 87:3-21

Gailis RM, Hill A (2006) A wind-tunnel simulation of plume dispersion within a large array of obstacles. Boundary-Layer Meteorol 119:289-338

Havens J, Walker H, Spicer TO (2001) Wind tunnel study of air entrainment into two-dimensional dense gas plumes at the Chemical Hazards Research Center. Atmos Environ 35:2305-2317

Högström U (1988) Non-dimensional wind and temperature profiles in the atmospheric surface layer: A re-evaluation. Q J R Meteorol Soc 99:680-687 
Hort MC, Robins AG (2002) The dispersion of fugitive emissions from storage tanks J Wind Eng Ind Aerodyn 90:1321-1348

Hunt JCR, Weber AH (1979) A Lagrangian statistical analysis of diffusion from a ground-level source in a turbulent boundary layer. Q J R Meteorol Soc 105:423443

Inagaki A, Kanda M (2008) Turbulent flow similarity over an array of cubes in near-neutrally stratified atmospheric flow. J Fluid Mech 615:101-120

Inagaki A, Kanda M (2010) Organized structure of active turbulence over an array of cubes within the logarithmic layer of atmospheric flow. Boundary-Layer Meteorol 135:209-228

Kanda I, Yamao Y (2011) Velocity adjustment and passive scalar diffusion in and above an urban canopy in response to various approach flows. Boundary-Layer Meteorol 141:415-441

Kanda I, Yamao Y, Uehara K, Ohara T (2011) A wind-tunnel study on diffusion from urban major roads. J Wind Eng Ind Aerodyn 99:1227-1242

Kanda I, Yamao Y, Uehara K, Wakamatsu S (2013)Particle-image velocimetry measurements of separation and re-attachment of airflow over two-dimensional hills with various slope angles and approach-flow characteristics. BoundaryLayer Meteorol 148:157-175

Leonardi S, Castro IP (2010) Channel flow over large cube roughness: a direct numerical simulation study. J Fluid Mech 651:519-539

Meade PJ (1959) The effects of meteorological factors on the dispersion of airborne material. Rassegns Internationale Electronica e Nucleare 6 Rassegna Rome, 11:107-130 
Nagata K, Sakai Y, Komori S (2001) Effects of small-scale freestream turbulence on turbulent boundary layers with and without thermal convection. Phys Fluids 23:065111

The NCAR Command Language (Version 6.0.0) [Software], Boulder, Colorado: NCAR/CISL/VETS, doi:10.5065/D6WD3XH5

Ogawa Y, Diosey PG, Uehara K, Ueda H (1981) A wind tunnel for studying the effects of thermal stratification in the atmosphere. Atmos Environ 15:807-821

Ogawa Y, Diosey PG, Uehara K, Ueda H (1982) Plume behavior in stratified flows. Atmos Environ 16:1419-1433

Ogawa Y, Diosey PG, Uehara K, Ueda H (1985) Wind tunnel observation of flow and diffusion under stable stratification. Atmos Environ 19:65-74

Ohba R, Hara T, Nakamura S, Ohya Y, Uchida T (2002) Gas diffusion over an isolated hill under neutral, stable and unstable conditions. Atmos Environ $36: 5697-5707$

Ohya Y (2001) Wind-tunnel study of atmospheric stable boundary layers over a rough surface. Boundary-Layer Meteorol 98:57-82

Ohya Y, Uchida T (2008) Laboratory and numerical studies of the atmospheric stable boundary layers. J Wind Eng Ind Aerodyn 96:2150-2160

Ohya Y, Uchida T (2004) Laboratory and numerical studies of the convective boundary layer capped by strong inversion. Boundary-Layer Meteorol $112: 223-240$

Ohya Y, Neff DE, Meroney RN (1997) Turbulence structure in a stratified boundary layer under stable conditions. Boundary-Layer Meteorol 83:139-161

Pasquill F (1961) The estimation of the dispersion of windborne material. Meteorol Magazine 90, No.1063 
Pope SB (2000) Turbulent Flows. Cambridge University Press, pp.771

Robins AG (1979) The development and structure of simulated neutrally stable atmospheric boundary layers. J Ind Aerodyn 4:71-100

Robins A (2003) Wind tunnel dispersion modelling some recent and not so recent achievements J Wind Eng Ind Aerodyn 91:1777-1790

Robins A, Castro I, Hayden P, Steggel N, Contini D, Heist D (2001a) A wind tunnel study of dense gas dispersion in a neutral boundary layer over a rough surface. Atmos Environ 35:2243-2252

Robins A, Castro I, Hayden P, Steggel N, Contini D, Heist D, Taylor TJ (2001b) A wind tunnel study of dense gas dispersion in a stable boundary layer over a rough surface. Atmos Environ 35:2253-2263

Robins A, Savory E, Scaperdas A, Grigoriadis D (2002) Spatial variability and source-receptor relations at a street intersection Water Air Soil Pollut: Focus $2: 381-393$

Sada K (2002) Wind tunnel experiment of tracer gas diffusion within unstable boundary layer over coastal region. Atmos Environ 36:4757-4766

Shirakata S, Nagai K, Mizumoto N (2002) Wind Tunnel Experiments for Atmospheric Diffusion under Various Stability Conditions. J Japan Soc Atmos Environ $37: 141-154$

Snyder WH (1981) Guideline for fluid modeling of atmospheric diffusion. Environmental Sciences Research Laboratory, U.S. Environmental Protection Agency, EPA-600/8-81-009

Snyder WH (2001) Wind-tunnel study of entrainment in two-dimensional densegas plumes at the EPA's fluid modeling facility. Atmos Environ 35:2285-2304 
Stull RB (1988) An introduction to boundary layer meteorology. Kluwer Academic Publishers, The Netherlands, pp.666

Uehara K, Murakami S, Oikawa S, Wakamatsu S (1997) Wind tunnel test of concentration fields around street canyons within the stratified urban canopy layer - Experimental studies on gaseous diffusion in urban areas Part 3 - J Archit Plann Environ Eng 499:9-16

Uehara K, Murakami S, Oikawa S, Wakamatsu S (1998) Wind tunnel evaluation of flow fields within street canyons with thermal stratification - Experimental studies on gaseous diffusion in urban areas Part 4 - J Archit Plann Environ Eng 510:37-44

Uehara K, Murakami S, Oikawa S, Wakamatsu S (1999) Wind tunnel experiments on how atmospheric stability and road width affect dispersion in urban street canyons - Experimental studies on gaseous diffusion in urban areas Part 5 - J Archit Plann Environ Eng 524:45-52

Uehara K, Hayashi S, Yamao Y, Matsumoto Y, Wakamatsu S (2007) Air pollution at a busy urban crossroad: estimation of annual mean NOx concentration distribution from field observations and wind-tunnel experiments. J Japan Soc Atmos Environ 42:93-106

Xie Z-T, Hayden P, Wood CR (2013) Large-eddy simulation of approaching-flow stratification on dispersion over arrays of buildings. Atmos Environ 71:64-74

Yassin MF (2013) A wind tunnel study on the effect of thermal stability on flow and dispersion of rooftop stack emissions in the near wake of a building. Atmos Environ 65:89-100

Yassin MF, Kato S, Ooka R, Takahashi T, Kouno R (2005) Field and wind-tunnel study of pollutant dispersion in a built-up area under various meteorological conditions. J Wind Eng Ind Aerodyn 93:361-382 
679 Yee E, Biltoft CA (2004) Concentration fluctuation measurements in a plume ${ }_{680}$ dispersing through a regular array of obstacles. Boundary-Layer Meteorol 681 111:363-415 